\title{
High-throughput screening in niche-based assay identifies compounds to target preleukemic stem cells
}

\author{
Bastien Gerby, ${ }^{1,2}$ Diogo F.T. Veiga, ${ }^{1,2}$ Jana Krosl, ${ }^{1}$ Sami Nourreddine, ${ }^{1,2}$ Julianne Ouellette, ${ }^{1,2}$ André Haman, ${ }^{1,2}$ Geneviève Lavoie, ${ }^{1,2}$ \\ Iman Fares, ${ }^{1}$ Mathieu Tremblay, ${ }^{1,2}$ Véronique Litalien, ${ }^{1}$ Elizabeth Ottoni, ${ }^{1,2}$ Milena Kosic,, ${ }^{1,2}$ Dominique Geoffrion, ${ }^{1,2}$ Joël Ryan, ${ }^{1}$ \\ Paul S. Maddox, ${ }^{3}$ Jalila Chagraoui, ${ }^{1}$ Anne Marinier, ${ }^{1}$ Josée Hébert, ${ }^{4}$ Guy Sauvageau, ${ }^{1,4}$ Benjamin H. Kwok, ${ }^{1,2}$ \\ Philippe P. Roux, ${ }^{1,2}$ and Trang Hoang ${ }^{1,2,5}$ \\ IInstitute for Research in Immunology and Cancer, and ${ }^{2}$ Molecular Biology Program, Faculty of Medicine, University of Montreal, Montreal, Quebec, Canada. ${ }^{3}$ Department of Biology, University of North \\ Carolina at Chapel Hill, Chapel Hill, North Carolina, USA. "4Lukemia Cell Bank of Quebec, Maisonneuve-Rosemont Hospital, Department of Medicine, and 5 Department of Pharmacology and Physiology and \\ Department of Biochemistry, Faculty of Medicine, University of Montreal, Montreal, Quebec, Canada.
}

Current chemotherapies for T cell acute lymphoblastic leukemia (T-ALL) efficiently reduce tumor mass. Nonetheless, disease relapse attributed to survival of preleukemic stem cells (pre-LSCs) is associated with poor prognosis. Herein, we provide direct evidence that pre-LSCs are much less chemosensitive to existing chemotherapy drugs than leukemic blasts because of a distinctive lower proliferative state. Improving therapies for T-ALL requires the development of strategies to target pre-LSCs that are absolutely dependent on their microenvironment. Therefore, we designed a robust protocol for high-throughput screening of compounds that target primary pre-LSCs maintained in a niche-like environment, on stromal cells that were engineered for optimal NOTCH1 activation. The multiparametric readout takes into account the intrinsic complexity of primary cells in order to specifically monitor pre-LSCs, which were induced here by the SCL/TAL1 and LMO1 oncogenes. We screened a targeted library of compounds and determined that the estrogen derivative 2-methoxyestradiol (2-ME2) disrupted both cellautonomous and non-cell-autonomous pathways. Specifically, 2-ME2 abrogated pre-LSC viability and self-renewal activity in vivo by inhibiting translation of MYC, a downstream effector of NOTCH1, and preventing SCL/TAL1 activity. In contrast, normal hematopoietic stem/progenitor cells remained functional. These results illustrate how recapitulating tissue-like properties of primary cells in high-throughput screening is a promising avenue for innovation in cancer chemotherapy.

\section{Introduction}

Current cancer chemotherapy targets proliferating and metabolically active cancer cells and efficiently reduces the tumor load. However, frequent relapses suggest the persistence of residual cancer stem cells that escaped treatment possibly due to a protective microenvironment that blunts their chemosensitivities (1-3). The reliance of cancer stem cells on their microenvironments and on non-cell-autonomous pathways (3) together with the failure of mainstream chemotherapy in a significant proportion of patients points to an urgent need for novel drug screening strategies designed to target cancer stem cells in their niche.

Recent evidence based on ultradeep sequencing of targeted genomic regions in acute leukemias revealed the existence of longlasting preleukemic hematopoietic stem cells within the bulk of leukemic cells that serve as a reservoir for disease progression (4, 5). While these cells are believed to resist therapy, direct evidence

Note regarding evaluation of this manuscript: Manuscripts authored by scientists associated with Duke University, The University of North Carolina at Chapel Hill, Duke-NUS, and the Sanford-Burnham Medical Research Institute are handled not by members of the editorial board but rather by the science editors, who consult with selected external editors and reviewers.

Conflict of interest: The authors have declared that no conflict of interest exists. Submitted: January 13, 2016; Accepted: September 22, 2016.

Reference information: / Clin Invest. 2016;126(12):4569-4584. doi:10.1172/JCI86489. for their distinctive chemosensitivities compared with leukemic blasts remains to be documented. In addition, treatment is limited by indiscriminate toxicity toward normal hematopoietic stem cells (HSCs). Therefore, the current challenge is to specifically target these preleukemic stem cells (pre-LSCs) that represent an extremely rare subpopulation in patients (4-7) and are not easily amenable to high-throughput screening (HTS) for novel drug discovery.

Acute lymphoblastic leukemia (ALL) is the most frequent pediatric cancer. T cell ALL (T-ALL), representing 15\%-20\% of ALL, can be classified into major molecular subgroups characterized by overexpression of oncogenic transcription factors (8, 9) that include $S C L / T A L 1$, a member of the basic helix-loophelix (bHLH) family, and LMO1 and LMO2, two LIM-only proteins (10). We and others have previously shown that oncogenic transcription factors in acute leukemia reprogram non-stem cell populations into aberrantly self-renewing pre-LSCs (7, 11-14). Using transgenic mouse models expressing the human $S C L$ and $L M O 1$ oncogenes in the thymus $(15,16)$, we showed that pre-LSCs remain dependent on physiological signaling from the microenvironment, specifically on activation of the NOTCH1/MYC axis (14). Our observations underscore the critical importance of a niche-based assay for pre-LSCs, to allow for the discovery of new classes of antileukemic drugs beyond those that have been identified so far using standard cell line screening. A challenge in HTS is the necessary miniaturization 
A

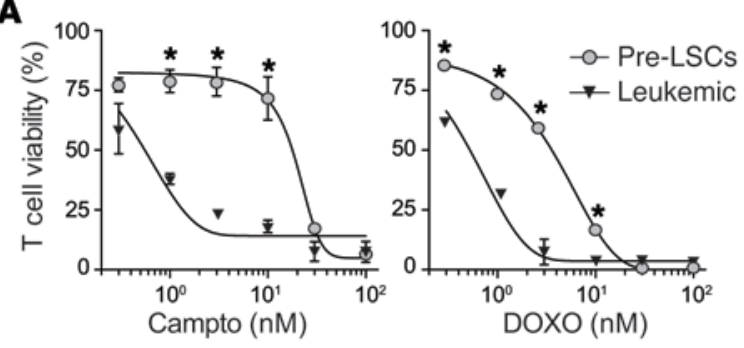

C

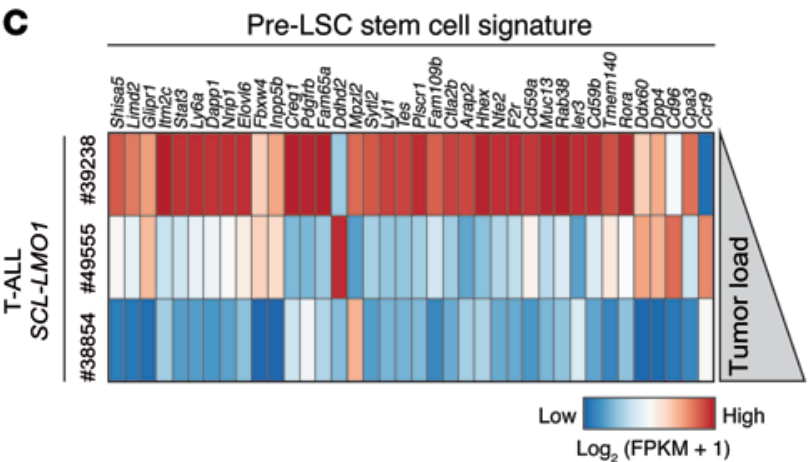

E

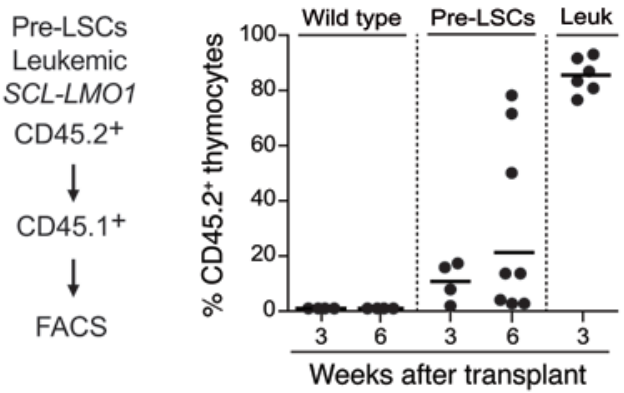

B
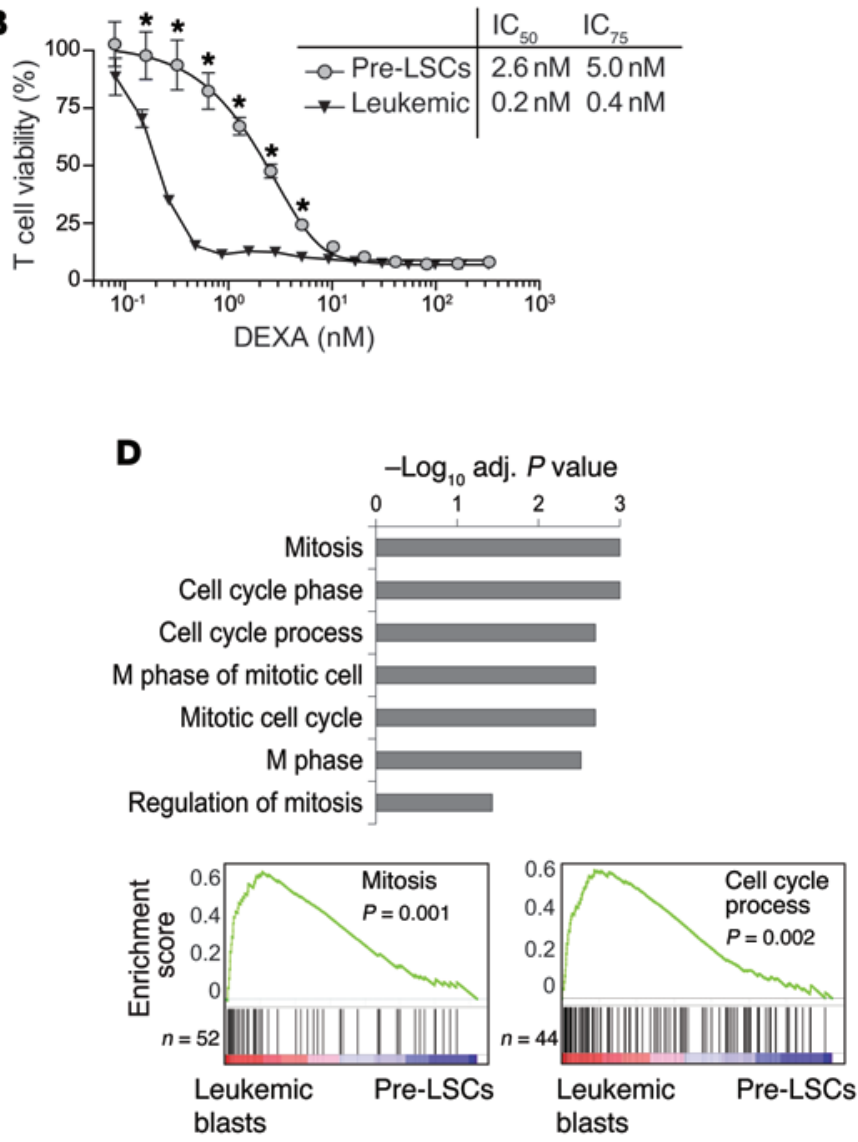

$\mathbf{F}$

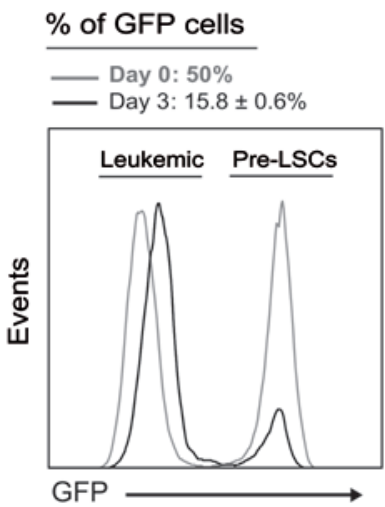

G

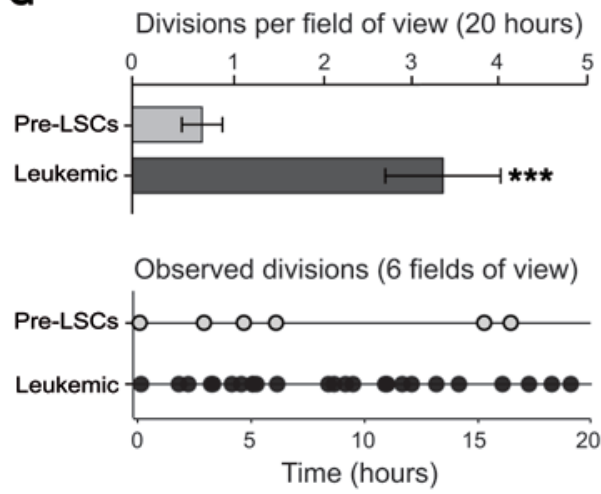

Figure 1. Proliferative leukemic blasts outcompete pre-LSCs and are more sensitive to chemotherapeutic agents. (A and B) Pre-LSCs are more resistant to the treatment than leukemic cells. Pre-LSCs or fully transformed SCL-LMO1 leukemic blasts were cocultured on OP9-DL1 stromal cells and treated with camptothecin (Campto, A, left panel), doxorubicin (DOXO, A, right panel), or dexamethasone (DEXA, B) for 3 days. Viable T cells were assessed by FACS (mean \pm SEM compared with controls, $n=6,{ }^{*} P \leq 0.05$ ). Representative of 2 independent experiments. (C) Inverse correlation between pre-LSC gene signature (14) and tumor load in Cd3 $e^{-/-S C L-L M 01 ~ T-A L L s . ~ D a t a ~ f r o m ~ R N A ~ s e q u e n c i n g ~ o f ~ C d 3 e ~} e^{-/-S C L-L M 01 T-A L L s ~ w e r e ~ r a n k e d ~ a c c o r d i n g ~ t o ~ t h e ~ t u m o r ~ l o a d ~(S u p p l e m e n t a l ~ F i g u r e ~ 1 A) . ~(D) ~ G e n e ~}$ set enrichment analysis for genes that are upregulated in leukemic blasts compared with pre-LSCs. Top panel: The top 7 gene ontology categories significantly enriched in T-ALL blasts (FWER $P<0.03)$. Bottom panel: Enrichment plots. (E) Pre-LSCs or leukemic blasts $\left(5 \times 10^{4}\right.$ cells, CD45.2) from Cd3e-l-SCL ${ }^{\text {tg }} L M O 1^{t g}$ mice or WT thymocytes were transplanted into recipient mice (CD45.1). Shown are the percentage of donor T cells $\left(\%\right.$ CD45.2 $2^{+}$Thy $\left.1.2^{+}\right)$per mouse analyzed 3 and 6 weeks later. Horizontal lines, median of thymic engraftment. (F and $\mathbf{C})$ Pre-LSCs exhibit low cell division frequency and are outcompeted by leukemic cells in vitro. SCLLM01 leukemic cells (GFP-) and pre-LSCs (GFP') were mixed in equal proportion and cultured for 3 days on OPg-DL1; GFP- and GFP' cells were analyzed before (gray curve) and after (black curve) coculture $(\mathbf{F}, n=3)$. The numbers of divisions per field of observation during 20 hours (G, top panel, $n=4)$ and each individual division observed at the indicated times in a total of 6 fields of view (G, bottom panel, $n=5-23$ ) were then compared between GFP- and GFP+ cells by time-lapse imaging (20 hours). ${ }^{* *} P \leq 0.001$. Representative of 2 independent experiments. 
A

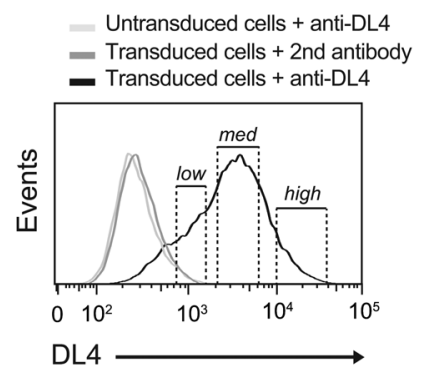

C

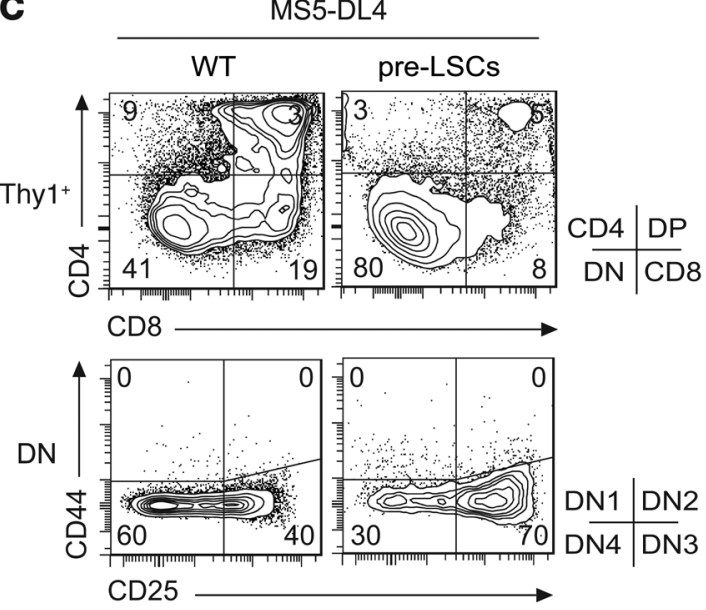

E

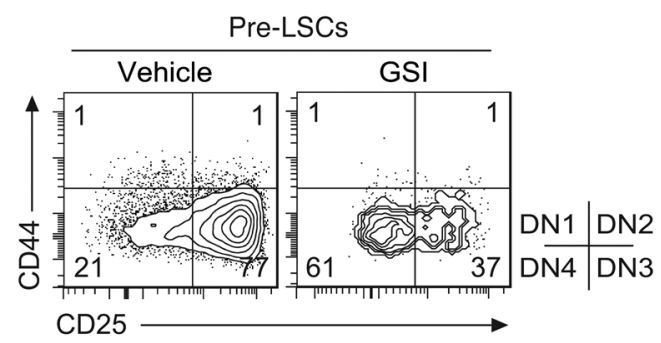

B
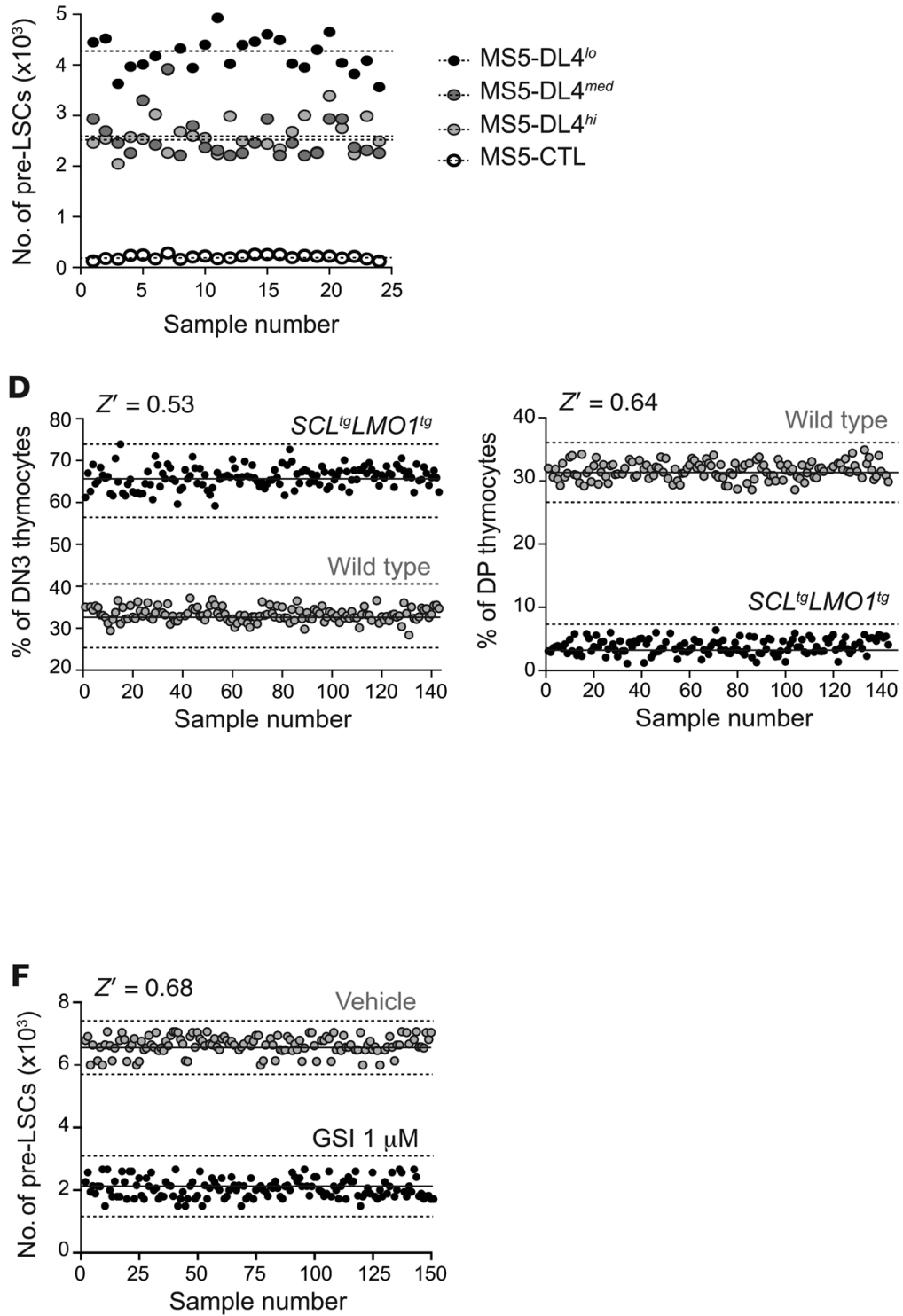

Figure 2. High-throughput assay for primary pre-LSCs in thymic-like microenvironment. (A and B) Generation of an optimal MS5-DL4 stromal cell line for pre-LSC viability. Following DL4 gene transfer, MS5 stromal cells were assessed for DL4 surface expression levels. Three MS5 subclones were purified according to levels of DL4 expression (low, medium, and high) (A). DN3 preleukemic SCL ${ }^{\text {tg }}$ LMO1 $^{\text {tg }}$ thymocytes were cocultured with MS5-CTL or MS5-DL4 ${ }^{\prime \prime}$, -DL4 ${ }^{\text {med }}$, or -DL4 ${ }^{\text {hi }}$ stromal cells during 5 days, and the absolute number of DN3 cells was assessed using FACS. Each dot represents an individual well, and dashed lines represent the mean for each condition (B). (C) Purified DN3 WT and preleukemic SCL ${ }^{\text {tg }} \mathrm{LMO}^{\text {tg }}$ thymocytes were cocultured with MS5-DL4 ${ }^{10}$ stromal cells. DN3-derived thymocytes were immunophenotyped 5 days later using the CD4, CD8, CD25, and CD44 markers. (D) Miniaturized coculture system for HTS-FACS in 384-well plate. The proportion of DN3 (left panel) and DP (right panel) thymocytes was then assessed using HTS-FACS, and assay reproducibility was calculated. Each dot represents an individual well. Solid lines and dashed lines represent the mean and 3 -fold the SD, respectively. The $Z^{\prime}$ factor is a statistical measure of the robustness of the HTS assay (ideally between 0.5 and 1 ). Representative of 3 independent experiments. (E and F) DN3 preleukemic SCL tg $L M O 1^{\text {tg }}$ thymocytes were cocultured with MS5-DL4 ${ }^{\text {lo }}$ stromal cells for 5 days in the presence or absence (Vehicle) of $\gamma$-secretase inhibitor (CSI, $1 \mu \mathrm{M}$ ), and the absolute number of DN3 cells was assessed using HTS-FACS. Representative FACS analysis of DN population is shown (E) together with assay reproducibility (F).

of a stromal-based coculture assay into a reproducible assay with a quantitative readout.

At the cellular level, these pre-LSCs serve as a reservoir for acquisition of Notch1 gain-of-function mutations (16) that recapitulate those identified in the human disease (17). Therefore, the mouse model closely mimics the leukemogenic process in humans, initially reported in monochorionic twins in which the initiating event is the establishment of an aberrant population of pre-LSCs found in both healthy and leukemic twins, with additional transforming event(s) in the leukemic patient (7). The identification of activating NOTCH1 mutations in over $60 \%$ of T-ALL patient samples in all molecular subgroups (17) rapidly led to the design of high-potency $\gamma$-secretase inhibitors (GSIs) targeting NOTCH1 signaling. GSIs are well tolerated with staggered dosing 
in several phase I clinical trials $(18,19)$. Nonetheless, efficient antileukemic activity might require sustained administration, which in turn could be a limiting factor (20). Interestingly, MYC is an essential downstream effector of NOTCH1 in T-ALL $(21,22)$, and decreasing $M y c$ expression via a BRD4 inhibitor effectively killed leukemia-initiating cells (23).

As discussed above, pre-LSCs are more genetically and phenotypically stable (14) than leukemia-propagating cells, which have acquired different sets of secondary mutations $(16,24)$. Importantly, we provided genetic evidence that self-renewal activity is a major determinant of leukemogenesis (14), further underscoring the importance of pre-LSC-based screening to identify novel antileukemic compounds. A major advantage of the mouse model is to provide unrestricted access to pre-LSCs (14).

Here, we demonstrate that pre-LSCs are much less sensitive to current treatment than the leukemic bulk, underscoring the importance of novel therapeutic development targeting pre-LSCs. We built on mechanistic insight gained in our previous work (14) to develop a robust protocol for a phenotypic screen with $S C L$ LMO1-induced pre-LSCs cultured within their niche-like microenvironment. Using FDA-approved compounds and drug-like small molecules, we identified 2-methoxyestradiol (2-ME2) as a new promising drug that abrogates pre-LSC viability and selfrenewal activity by inhibiting MYC translation and SCL accumulation, thereby antagonizing cell-autonomous as well as non-cellautonomous pathways.

\section{Results}

Pre-LSC low chemosensitivity and low proliferation rate. Pre-LSCs are believed to be chemoresistant and to drive relapse $(4,24)$, although direct evidence for differential chemosensitivity between leukemic blasts and pre-LSCs remains to be documented. We took advantage of genetic mouse models to directly address the distinctive properties of pre-LSCs compared with fully transformed cells using in vitro and in vivo approaches. We previously showed that $C d 3 e^{+/+}$or $C d 3 e^{-/-} \mathrm{DN} 3$ thymocytes are the only pre-LSCenriched population in mice expressing SCL-LMO1 (14), hereafter "pre-LSCs." First, we compared the response of pre-LSCs and T-ALL blasts to 2 commonly used inhibitors of DNA replication, doxorubicin and camptothecin (Figure 1A). Dose-response curves indicate that leukemic cells are 10- to 20-fold more sensitive to the 2 drugs than pre-LSCs in vitro. Moreover, this difference was also observed with dexamethasone (DEXA; Figure 1B), a glucocorticoid often used for stratification and treatment of childhood and adult T-ALL (25). We conclude that pre-LSCs are poorly responsive to current chemotherapeutic drugs.

We next analyzed by RNA sequencing the transcriptomes of 3 SCL-LMO1 T-ALLs taken at different stages of leukemia development, as assessed by the tumor load. Strikingly, we found that the pre-LSC stem cell gene signature (14) was inversely correlated with the tumor load, supporting the view that pre-LSCs were progressively outcompeted by more aggressive leukemic cells (Figure 1C and Supplemental Figure 1A; supplemental material available online with this article; doi:10.1172/JCI86489DS1). Conversely, the top gene ontology categories associated with acute leukemia compared with the preleukemic stage are mitosis and cell cycle (Figure 1D), indicating that leukemic blasts are more proliferative than pre-LSCs, hence their higher sensitivities to topoisomerase inhibitors. The enrichment in cell cycle genes was manifested in vivo by the kinetic of thymic reconstitution after transplantation into sublethally irradiated congenic recipient mice (Figure 1E). Within 3 weeks, T-ALL blasts rapidly and massively invaded the thymus (Figure 1E), spleen, and BM (Supplemental Figure 1B), while pre-LSCs induced a modest and heterogeneous thymic reconstitution at 3 weeks after transplantation and even at 6 weeks, without engrafting other organs. As expected, WT thymocytes did not engraft (Figure 1E and Supplemental Figure 1B). To directly address the question of whether leukemic blasts are more competitive than self-renewing pre-LSCs, we set up an equal-split competition in which equal proportions $(50 \%)$ of $\mathrm{GFP}^{+}$pre-LSCs from SIL-SCL Lck-LMO1 double-transgenic mice (SCL $L^{\text {tg } L M O 1^{t g}}$ mice), which express the human SCL and LMO1 genes, and nonGFP leukemic cells were cultured on OP9-DL1 (Figure 1F). Within 3 days, T-ALL blasts outcompeted pre-LSCs in culture as observed by the 3 -fold reduction of the proportion of $\mathrm{GFP}^{+}$pre-LSCs (Figure $1 F)$. Furthermore, time-lapse imaging (Supplemental Figure 1C) of this equal-split competitive assay performed 6 hours after plating revealed that the division rate of leukemic cells was 4 times higher than that of pre-LSCs (Figure 1G).

Together, our results demonstrate that pre-LSCs have a low proliferative status and are more resistant than leukemic cells to current chemotherapeutic drugs that target proliferating cells, such as doxorubicin. Based on the evidence that pre-LSCs caused relapse (6), these cells represent the prime target for new drug development.

Design of a high-throughput screening protocol based on pre-LSC viability in a niche-like microenvironment. Several essential parameters needed to be integrated into the design of a robust pre-LSCbased assay for high-throughput screening (HTS). First, pre-LSCs are extremely rare in leukemic patient samples, requiring extensive purification by flow cytometry and ultradeep sequencing to reveal mutations that occur in these cells $(4,24)$. This is because pre-LSCs are much less competitive than leukemic blasts, as shown above, and are highly diluted in the leukemic mass at the time of overt leukemia. We took advantage of the preleukemic stage in transgenic mouse models to have unrestricted access to a reproducible source of pre-LSCs. These cells were harvested at weaning, long before the acquisition of secondary mutations and leukemia onset (16). Second, we opted for a phenotypic screen for several reasons. Given the molecular diversity of T-ALL, we reasoned that a phenotypic screen using primary pre-LSCs has a higher likelihood of identifying small-molecule inhibitors of selfrenewal, rather than targeting the diversity of oncogenes in the 5 molecular subgroups of T-ALL $(10,26)$. In addition, pre-LSCs in 3 different genetic models of T-ALL of the bHLH subgroup are DN3 cells $(12,14,27)$, and rely on the same self-renewal genes for maintenance in vivo $(12,14,27)$, reinforcing the suitability of this population as target. Moreover, a multiparametric phenotypic screen takes into account the heterogeneity of primary cells $(14,28)$ and allows for a quantitative assessment of this specific subset of cells based on surface phenotype.

Finally, we previously showed that pre-LSCs conserve their dependence on the thymic microenvironment and require coculture on OP9 stromal cells expressing delta-like 1 (DL1) for survival in culture (14), much like their normal counterparts (29). We 
A

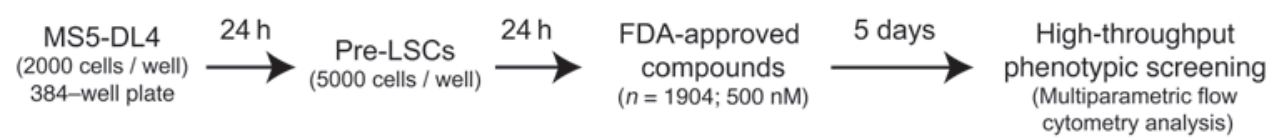

B
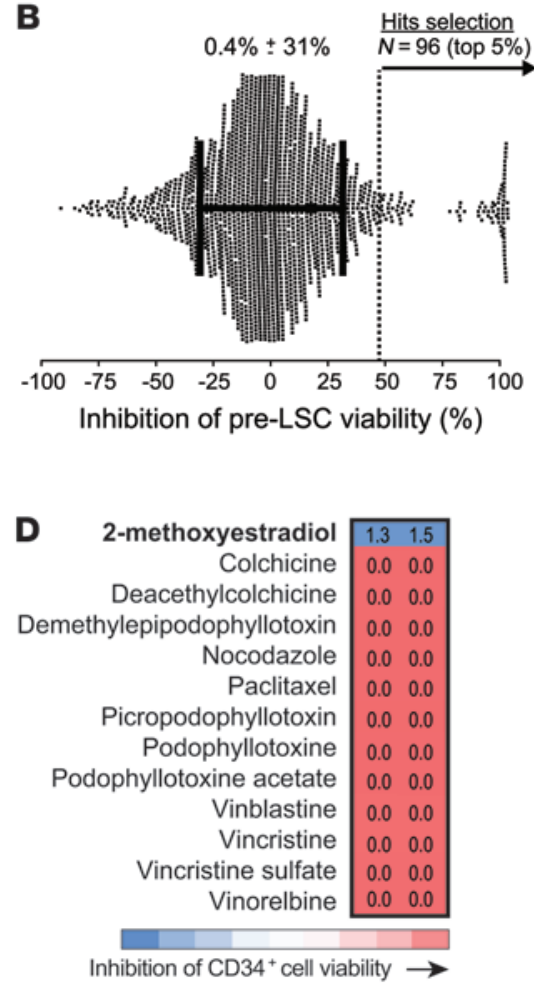

$\mathbf{F}$

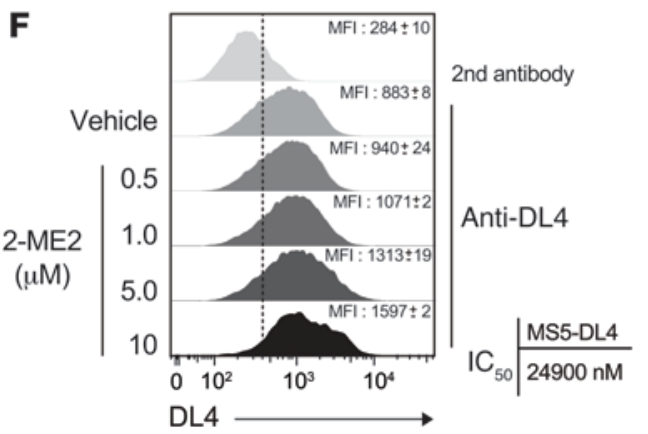

C

E
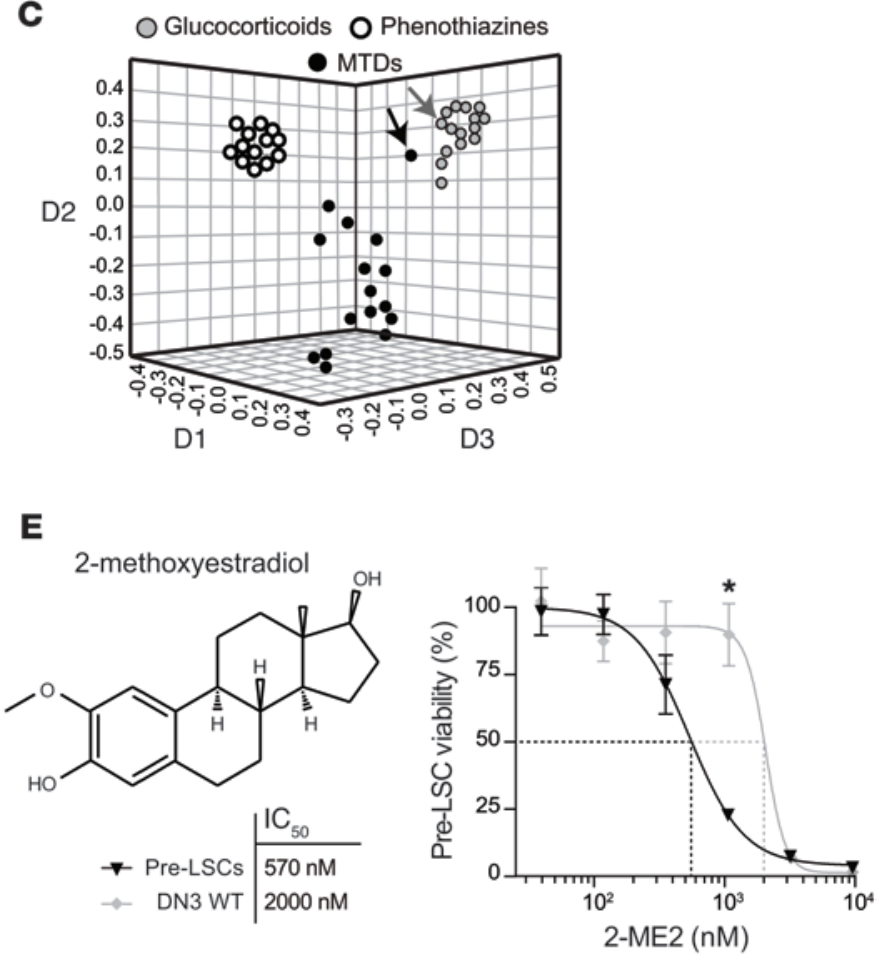

G

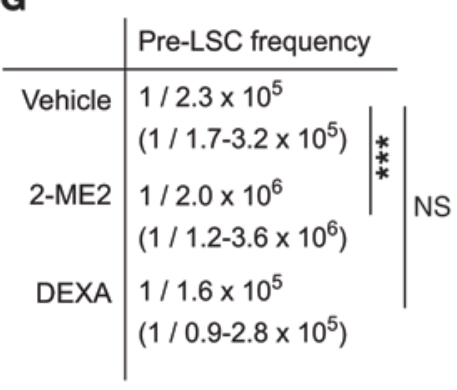

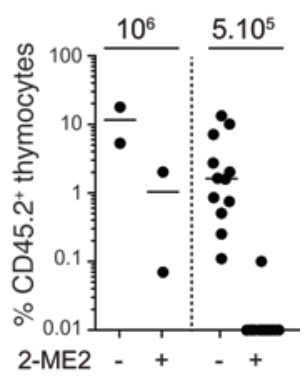

Figure 3. Small-molecule screening identifies 2-ME2 as an inhibitor of pre-LSCs. (A) Experimental procedure for chemical screening of pre-LSCs. (B) The inhibition of pre-LSC viability was calculated for each compound (CSI set as $100 \%$ and DMSO as $0 \%$; average pre-LSC viability: $0.4 \%$ ). The top $5 \%$ ( $n=96$ ) of hits were selected. (C) Clustering of 44 selected compounds by structural similarities using the ChemMine tool (http://chemmine.ucr.edu/). The multidimensional scaling algorithm maps a compound described by its chemical structure into a 3D space and where neighbor compounds exhibit similar molecular structure. The axes (D1, D2, and D3) refer to the dimensions generated by the multidimensional scaling. Black arrow identifies 2-ME2 and gray arrow DEXA. MTDs, microtubuletargeting drugs. (D) 2-ME2 is the only MTD that does not affect the viability of human HSCs. Human CD34+ peripheral blood was collected from cord blood cells and treated with the indicated MTDs at $1 \mu$ M during 1 week as described previously (38). Numbers $(n=2)$ represent the fractions of viable cells compared with vehicle control. (E) 2-ME2 structure (top left panel) and dose-response on purified pre-LSCs and DN3 WT thymocytes. The percentages of cell viability are shown (right panel, $n=6,{ }^{*} P \leq 0.05$ ). IC $\mathrm{C}_{50}$ was calculated (bottom left panel). Representative of 2 independent experiments. (F) MS5-DL4 stromal cells alone were

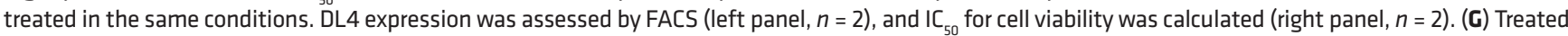
and untreated viable cells were then transplanted by limiting dilution assay into recipient mice according to Supplemental Figure $5 \mathrm{~A}$. Thymic engraftment was analyzed 6 weeks after transplantation, and pre-LSC frequencies were calculated (left panel) by Poisson statistics (Cls in parentheses; $\left.{ }^{* * *} P \leq 0.001\right)$. Right panel: Thymic reconstitution by control or 2-ME2-treated cells.

therefore designed a miniaturized coculture assay for pre-LSC viability in their "habitat." To this end, we replaced OP9 cells, which need replating every 3 days, with MS5 stromal cells, which can be maintained for at least 7 days in culture (30) and are more appropriate for a robust chemical HTS assay that requires several days of drug exposure. We generated MS5 stromal cell lines expressing different levels of the most physiological NOTCH1 ligand, delta-like 4 (DL4) $(31,32)$, for coculture with $S C L^{t g} L M O 1^{\text {tg }}$ 

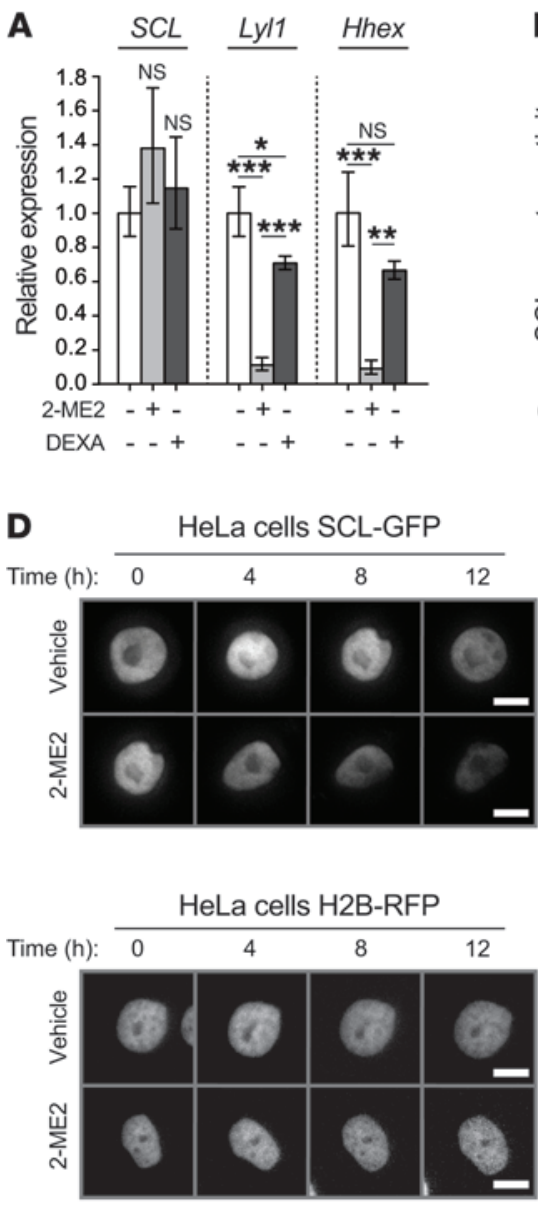

H

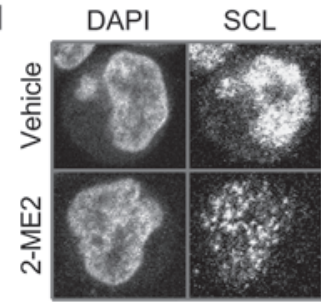

B
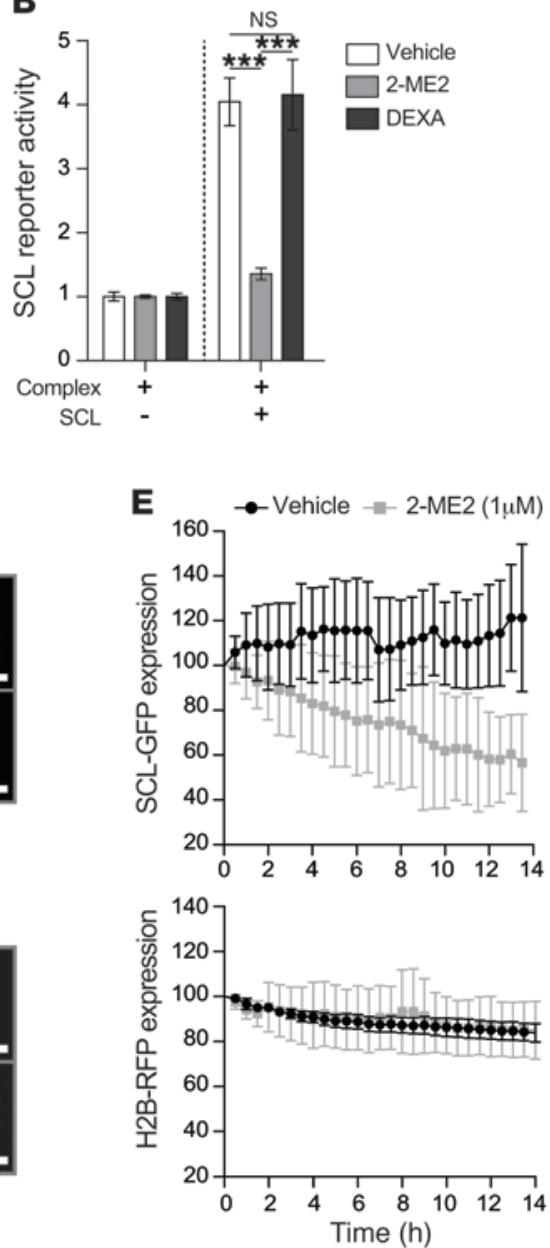

C

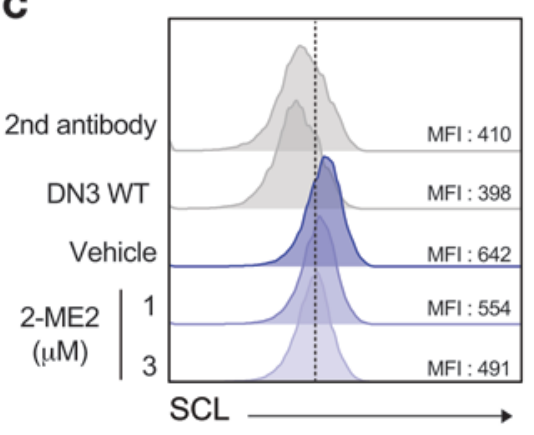

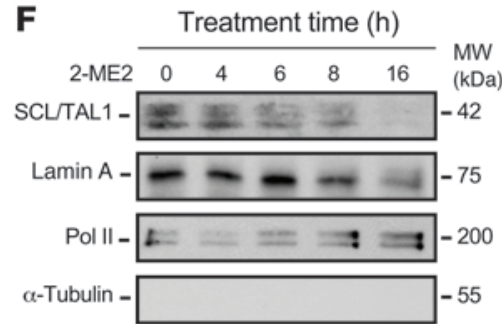

G

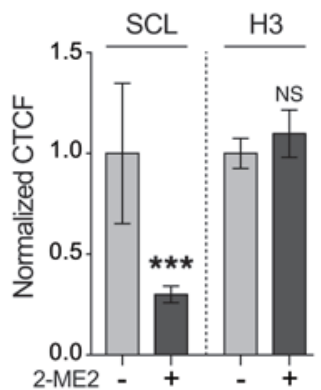

Figure 4. 2-ME2 inhibits the accumulation of SCL protein. (A) Pre-LSCs from SCL ${ }^{\text {tg }} L M O 1^{\text {tg }}$ thymocytes were treated with 2-ME2 (1 $\mu M$ ) or DEXA (5 nM) for 16 hours, and mRNA levels of SCL and SCL target genes Ly/1 and Hhex were determined by quantitative RT-PCR and normalized to $\beta$-actin (mean \pm SD, $n=3,{ }^{*} P \leq 0.05,{ }^{*} P \leq 0.01,{ }^{* *} P \leq 0.001$ ). (B) $2-\mathrm{ME} 2$ inhibits the activity of the SCL transcriptional complex. The Gypa promoter (Gypa-Luc) was used as a reporter. Transfected cells were then treated or not (Vehicle) with 2-ME2 ( $1 \mu \mathrm{M})$ or DEXA ( $2 \mathrm{nM})$ for 24 hours. Results are expressed as fold activation by SCL over the complex without SCL. Data represent the mean $\pm \mathrm{SD}\left(n=3,{ }^{* *} P \leq 0.001\right)$. (C) Immunofluorescence of human SCL oncoprotein by flow cytometry in pre-LSCs from SCL ${ }^{\text {tg }} L M 01^{\text {tg }}$ thymocytes treated with the indicated doses of 2-ME2 or not (Vehicle) for 16 hours. DN3 WT thymocytes stained with antibody against SCL and cells stained with the secondary antibody only were used as negative controls. (D and E) Real-time imaging of HeLa cells expressing SCL-CFP or H2B-RFP in the presence or absence of 2-ME2 $(1 \mu \mathrm{M})$. Kinetic of GFP (top panels) and RFP (bottom panels) expression in a representative treated or untreated cell is shown (D), and the averages of mean fluorescence intensities (MFIs) for each time point were calculated (E). MFIs were normalized to the initial intensity of the corresponding cell, which was set as $100 \%$. (F) JURKAT cells were treated with 2 -ME2 $(1 \mu \mathrm{M})$ for the indicated times, and nuclear extracts were subjected to immunoblotting. Pol II, RNA polymerase II. (G and H) SCL and histone H3 intracellular immunofluorescence staining in JURKAT cells treated or not with 2-ME2 $(1 \mu \mathrm{M})$ for 8 hours. The normalized corrected total cell fluorescence (CTCF) per nucleus of SCL $\left(n=27-98,{ }^{* *} P \leq 0.001\right)$ and H3 $(n=42-60)$ was calculated (G). A representative example of SCL (left panel) and H3 (right panel) expression is shown $(\mathbf{H})$. All data shown are representative of 2 independent experiments.

pre-LSCs (Figure 2A). We found that MS5-DL4 ${ }^{l o}$ cells sustained viability of $S C L^{t g} L M O 1^{t g}$ pre-LSCs more efficiently than MS5 expressing higher DL4 levels (Figure 2B). We therefore selected MS5-DL4 ${ }^{\text {lo }}$ (hereafter "MS5-DL4") for further experiments.
These coculture conditions favored the differentiation of purified WT DN3 thymocytes to the double-positive (DP) stage within 5 days (Figure 2C). In contrast, SCL-LMO1-expressing pre-LSCs remained blocked at the DN3 stage during the same time frame 
A

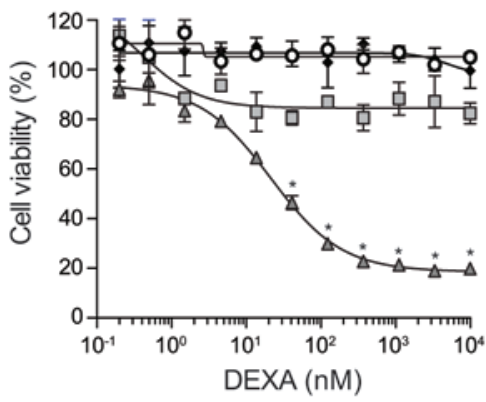

D

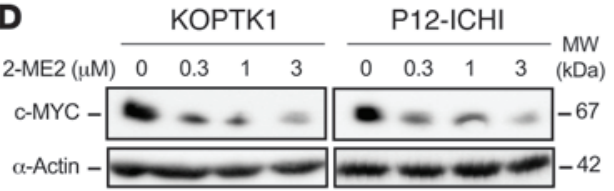

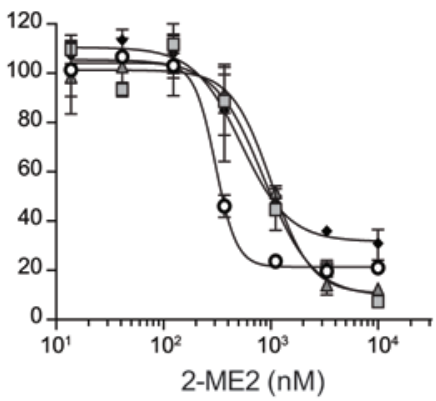

B

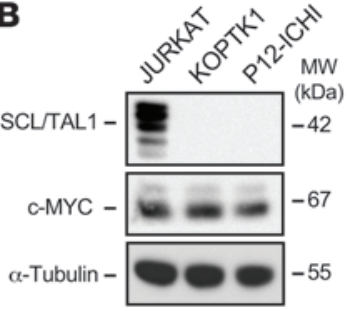

C

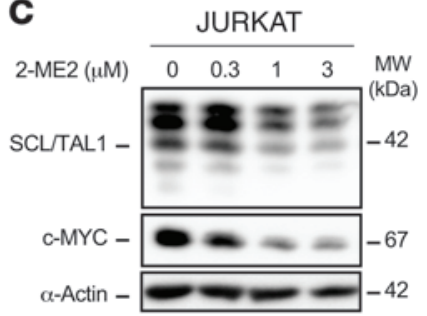

E

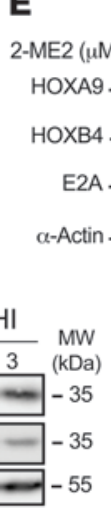

$\mathbf{F}$

\begin{tabular}{l|cc} 
F & \multicolumn{2}{c}{$\mathrm{t}_{1 / 2} \mathrm{SCL}(\mathrm{h})$} \\
\cline { 2 - 3 } & Vehicle & 2-ME2 \\
\hline JURKAT & 11.0 & 10.2
\end{tabular}

\begin{tabular}{c|cc} 
& \multicolumn{2}{c}{$\mathrm{t}_{1 / 2} \mathrm{C}-\mathrm{MYC}(\mathrm{h})$} \\
\cline { 2 - 3 } & Vehicle & $2-\mathrm{ME} 2$ \\
\hline JURKAT & 1.7 & 1.8 \\
KOPTK1 & 4.9 & 4.5
\end{tabular}

G

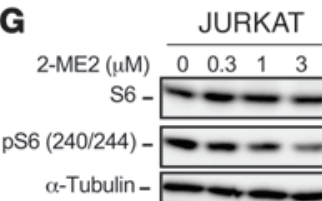

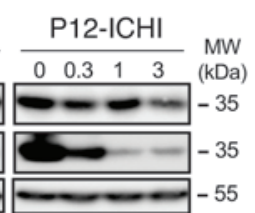

H

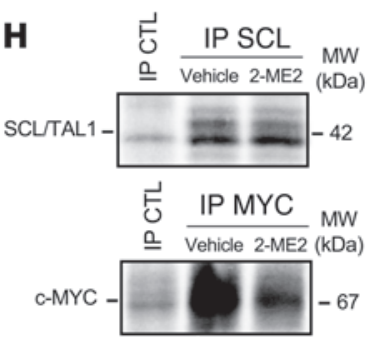

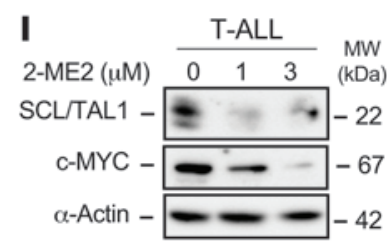

Figure 5. 2-ME2 inhibits glucocorticoid-resistant T-ALL cells and targets MYC protein synthesis. (A) Dose-response curves of DEXA (left panel) and 2-ME2 (right panel) of 4 human T-ALL cell lines (JURKAT, KOPTK1, P12-ICHIKAWA, and HPB-ALL) treated for 48 hours (mean \pm SEM, $n=3,{ }^{*} P \leq 0.05$ ). Cell viability was assessed by FACS (percent control). (B) Levels of SCL and c-MYC protein expression were determined by Western blot in the 3 indicated T-ALL cell lines after total protein extraction. (C-E) JURKAT, KOPTK1, and P12-ICHIKAWA cells were treated for 16 hours with the indicated doses of 2-ME2. Nuclear protein extracts were subjected to immunoblotting with the indicated antibodies. $\alpha$-Actin was used as a loading control. (F) SCL and c-MYC half-lives were determined in JURKAT (SCL+c-MYC+) and KOPTK1 (c-MYC+) T-ALL cells by cycloheximide chase and Western blotting according to Supplemental Figure 9D. Representative of 2 independent experiments. (G) JURKAT, KOPTK1, and P12-ICHIKAWA cells were treated for 16 hours with the indicated doses of 2-ME2. Total protein extracts were subjected to immunoblotting with the indicated antibodies. $\alpha$-Tubulin was used as a loading control. Representative of 2 independent experiments. (H) JURKAT cells were treated with 2-ME2 (3 $\mu \mathrm{M})$ or not (Vehicle) during 3 hours in the presence of ${ }^{35} \mathrm{~S}$-methionine. SCL (top panel) and c-MYC (bottom panel) immunoprecipitation was then performed on labeled proteins. Immunoglobulins were used as control of immunoprecipitation (IP CTL). Radiolabeling was revealed using a Phosphorlmager. (I) T-ALL blasts from a thymoma of SCL ${ }^{\text {tg }} L M O 1^{\text {tg }}$ mice were treated for 16 hours with the indicated doses of 2-ME2. The human SCL transgene and endogenous c-MYC protein expression levels were then determined by immunoblotting. $\alpha$-Actin was used as a loading control. Representative of 2 independent experiments.

(Figure 2C), reproducing the differentiation arrest observed in vivo $(33,34)$. Similar results were observed when the assay was miniaturized in 384-well format (Figure 2D). Finally, as proof of concept for the screen, cultures were exposed to GSI at $1 \mu \mathrm{M}$ or to the vehicle alone (Figure 2, E and F). The drug was added 12 hours after seeding to allow for pre-LSC-stromal interaction before treatment. GSI strongly and reproducibly killed pre-LSCs as reported (14), with a robust $Z$ ' factor. By taking into account the heterogeneity of primary cells $(14,28)$ and the necessity of nichelike microenvironments (14), the designed phenotypic screen allows for a quantitative assessment of pre-LSCs. We therefore conclude that the assay is suitable for HTS.

Small-molecule screening and identification of an inhibitor of preLSC self-renewal activity. Using the above protocol, we screened a library of 1,904 compounds on pre-LSC viability (Figure 3, A and
B, and Supplemental Figure 2). GSI (14) served as an internal reference for the screen (Figure 3B and Supplemental Figure 2). The majority of tested compounds had no effect on pre-LSC viability, underscoring the selectivity of the assay and the specificity of the hits. We selected the top $5 \%$ of hits that reduced the viability of pre-LSCs (Figure 3B, range of 45\%-100\% inhibition). While our assay conditions enable the identification of potential inducers of differentiation (Figure 2D and Supplemental Figure 2), no compound was found to overcome the DN to DP differentiation blockade imposed by SCL-LMO1. We then applied 3 criteria to filter the viability hits. First, compounds were clustered according to structural similarities (http://chemmine.ucr.edu/). Second, we determined compound selectivity by comparing our results with those obtained in a screen of 20 primary acute myeloid leukemia (AML) specimens (Supplemental Table 1), based on the premise that pri- 
A

T-ALL

SCL-LMO1

CD45.2+

(donor)

$\downarrow$

CD45.1+

(host)

I

$\mathrm{BM}$ aspiration (S10C)

$\downarrow$

Vehicle or 2-ME2

(Osmotic pumps; $40 \mathrm{mg} / \mathrm{kg} /$ day) $\downarrow$

$\operatorname{FACS}(\mathrm{B}, \mathrm{C}, \mathrm{E})$

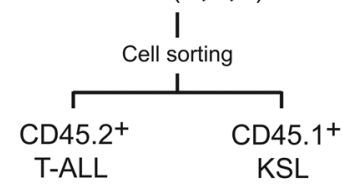

$\bigsqcup_{\substack{\text { T-ALL } \\ \text { Survival (D) }}}^{\text {Transplant }}$
B
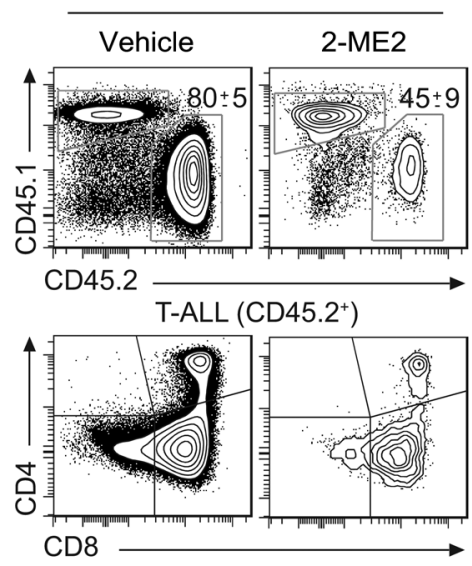

Normal cells (CD45.1 $\left.1^{+}\right)$

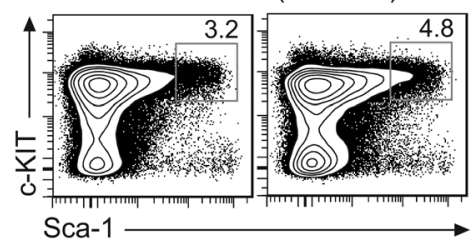

C

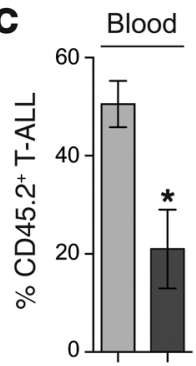

2-ME2 - + 2-ME2
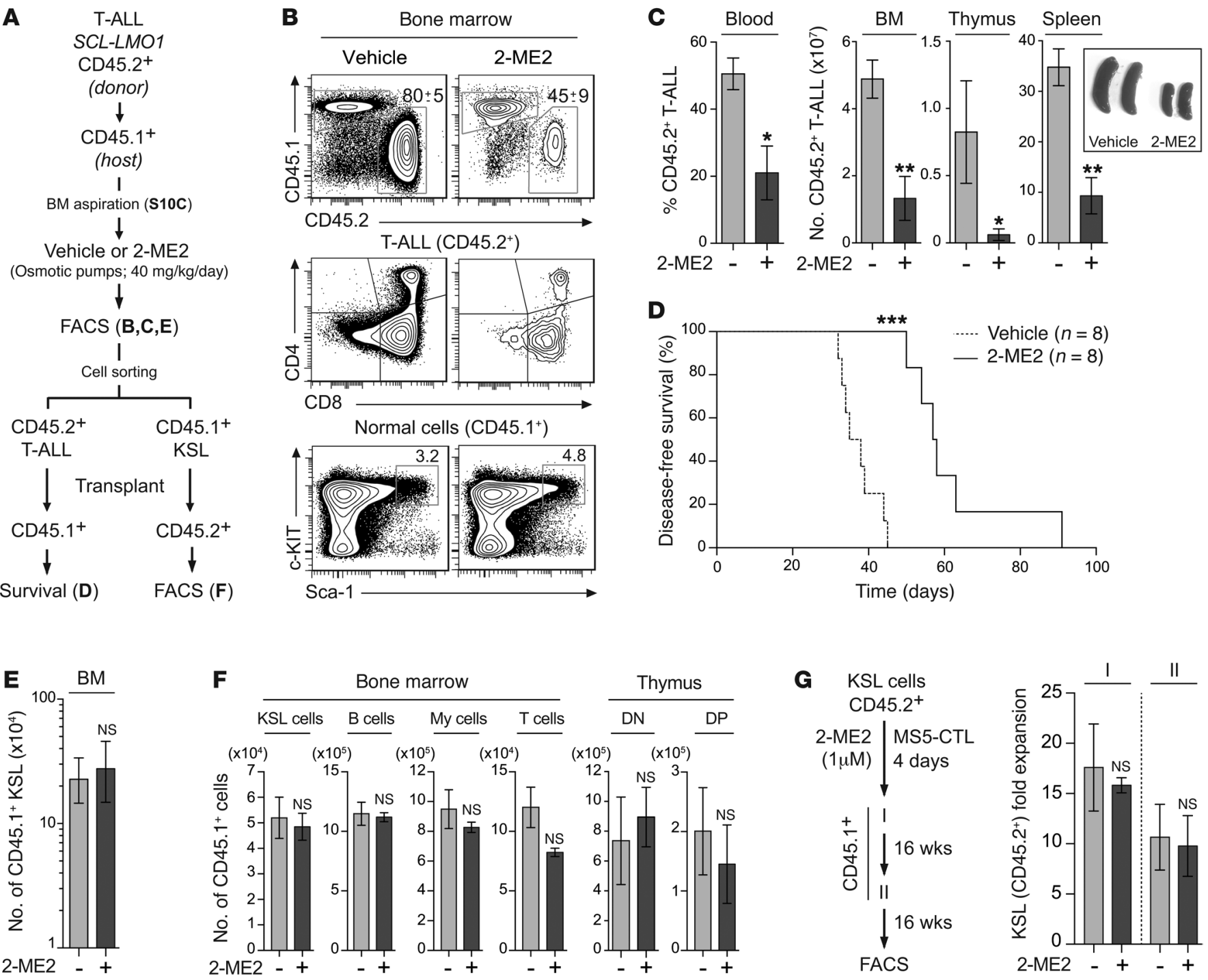

Figure 6. 2-ME2 inhibits T-ALL development without affecting HSC functions in vivo. (A) Experimental procedure to study the in vivo effect of 2-ME2 on T-ALL development and HSC functions in a preclinical model. (B) Representative FACS profiles of CD45.2 (donor) and CD45.1 (host) expression in the BM cells of mice treated or not (Vehicle) with 2-ME2 (top panel). The expression of CD4/CD8 and c-Kit/Sca-1 markers is shown in CD45.2+ T-ALL cells (middle panel) and CD45. $1^{+}$lineage- cells (bottom panel), respectively. (C) The proportion of T-ALL blasts (CD45.2+) was assessed in the blood ( $\left.n=5\right)$. Absolute numbers of T-ALL blasts (CD45.2+) from the BM, thymus, and spleen were calculated, and pictures of spleens are shown (right panel, $\left.n=5,{ }^{*} P \leq 0.05,{ }^{* *} P \leq 0.01\right)$. (D) Kaplan-Meier survival curve of secondary CD45.1+ mice transplanted with T-ALL blasts from 2-ME2-treated and untreated primary recipient (***P $\leq 0.001$ ). (E) Absolute numbers of HSC-enriched populations (KSL) from the BM of recipient mice (CD45.1 $)$ were calculated after treatment $(n=5)$. (F) Absolute numbers of donor-derived CD45.1 $\mathrm{HSCs}(\mathrm{KSL})$ and B, myeloid (My), and T cells in the BM of secondary CD45.2 $2^{+}$recipient mice were calculated 14 weeks after transplantation $(n=5)$. Absolute numbers of donor-derived CD45.1+ thymocytes (DN and DP) were calculated in the thymus $(n=5)$. There was no significant difference between controls and 2-ME2-treated conditions. (C) KSL cells from the BM of CD45.2+ mice were purified and treated or not with 2-ME2 (1 $\mu$ M) on MS5-CTL cells during 4 days. Treated and untreated cells were then transplanted into primary and secondary lethally irradiated CD45.1 $1^{+}$mice (left panel, $n=3-5)$. The fold expansions of CD45.2 $2^{+}$KSL cells in the BM were calculated after primary and secondary transplantation. There was no significant difference between controls and 2-ME2-treated conditions.

mary human AML cells are significantly different from T-ALL. Third, we searched for compounds that spare normal hematopoietic stem/progenitor cells (HSPCs).

Among the viability hits, 42 compounds clustered in 3 major groups (Figure 3C and Supplemental Table 2). The first group included 15 glucocorticoids, well-known inducers of thymocyte apoptosis (Figure 3C and Supplemental Table 2), in particular DEXA, which is currently used in T-ALL treatment. The second group included compounds of the phenothiazine family (Figure $3 \mathrm{C}$ and Supplemental Table 2) that were recently identified in a screen on MYC-induced T-ALL in zebrafish (35), demonstrating the convergence of 2 functional screens, 1 based on whole-animal and the other on organotypic cultures of primary cells. Interestingly, glucocorticoids and phenothiazines with the exception of perphenazine (35) mostly spared the AML samples that were tested (Supplemental Figure 3), indicating the discriminatory power 
A

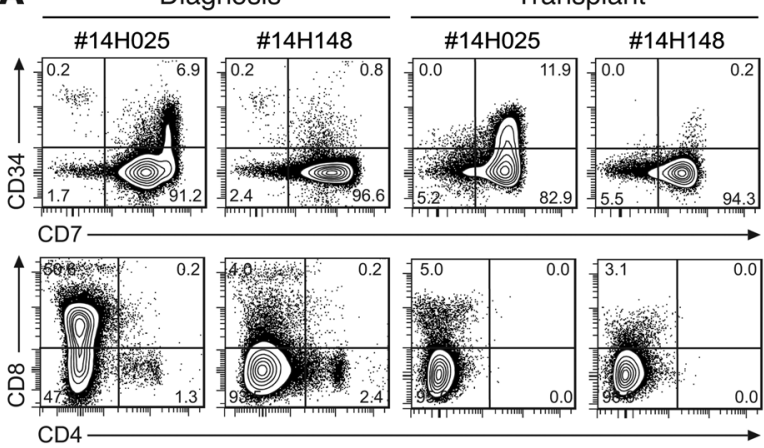

B

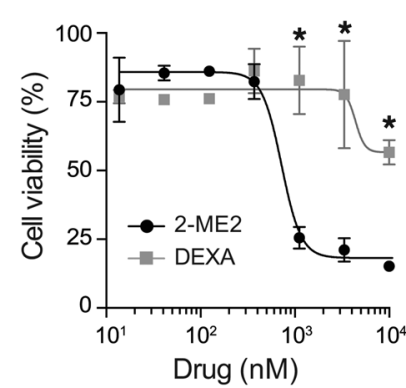

\#14H148

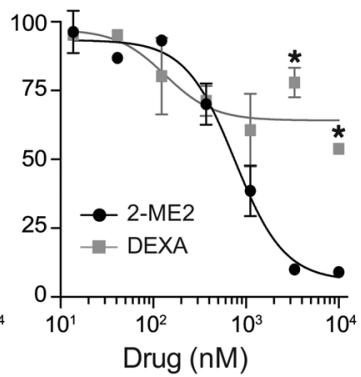

C

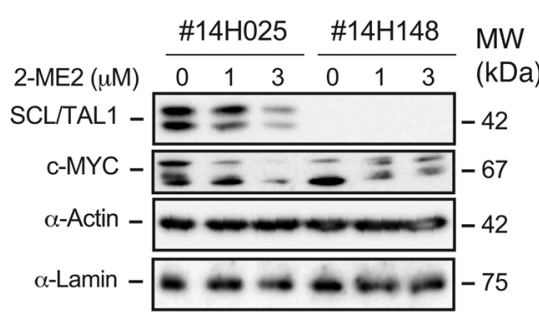

E

Human T-ALL
$\# 14 \mathrm{H} 025$
$\# 14 \mathrm{H} 148$
$\downarrow$
$\mathrm{NSG}$ mice
$\square$
BM aspiration
(right panel)
$\downarrow$
Vehicle
or 2-ME2
$\downarrow$
FACS (F)

D

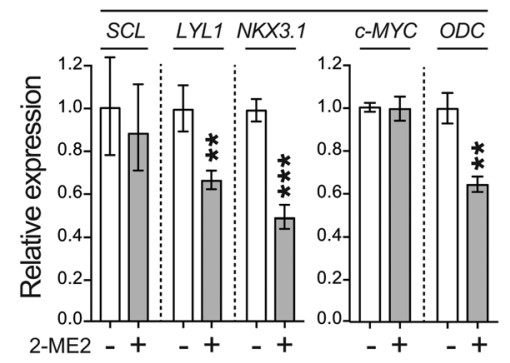

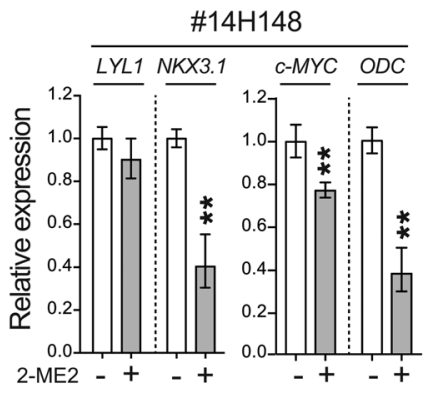

$\mathbf{F}$

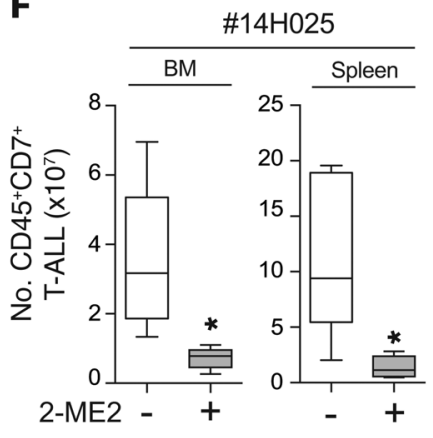

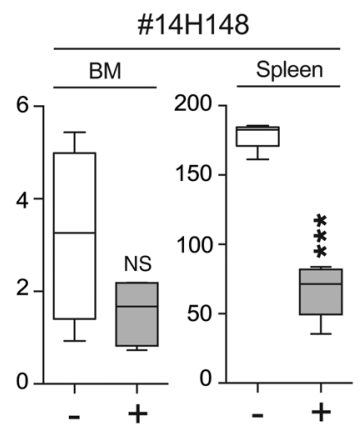

Figure 7. 2-ME2 reduces human T-ALL development in xenograft models. (A) Immunophenotype of human T-ALL samples (nos. 14H025 and 14H148) at diagnosis and after transplantation in NSG mice. The expression of human CD34/CD7 and CD4/CD8 markers of peripheral blood cells from the patients (left panel) and of engrafted human CD45+ T-ALL blasts in the BM of NSG mice 6 weeks after transplantation (right panel) is shown. (B) Purified engrafted human CD45+CD7 ${ }^{+}$ T-ALL blasts from patients $14 \mathrm{HO} 25$ and $14 \mathrm{H} 148$ were cocultured on MS5-DL4 stromal cells with a dose-response of $2-\mathrm{ME2}$ and DEXA for 4 days $\left(n=3\right.$, ${ }^{*} P \leq 0.05$ ). The percentages of cell viability are shown for both patients. (C) Blasts from both patients were treated with the indicated doses of 2-ME2 for 16 hours. SCL and c-MYC protein expression levels were determined by immunoblotting. (D) Blasts were treated with $1 \mu \mathrm{M}$ of 2-ME2 during 16 hours, and mRNA expression levels of the indicated genes were assessed by quantitative RT-PCR and were normalized to $\beta$-actin mRNA $\left(n=3,{ }^{* *} P \leq 0.01,{ }^{* * *} P \leq 0.001\right)$. (E) Experimental procedure to study the effect of 2-ME2 on human T-ALL in vivo (left panel). Engrafted T-ALL blasts of both patients were transplanted into secondary NSG mice $(n=10)$, and the proportion of $C D 45^{+} C D 7^{+} T-A L L$ blasts in BM aspirations was assessed 3 weeks after transplantation (right panel). (F) Engrafted secondary mice were randomly selected for treatment or not (Vehicle, $n=5$ ) with 2-ME2 (40 mg/kg/d, $n=5$ ) using osmotic pumps during 7 days. Human T-ALL reconstitution (number of $\mathrm{CD} 45^{+} \mathrm{CD} 7^{+}$blasts) was monitored by FACS in the BM and the spleen $\left({ }^{*} P \leq 0.05,{ }^{* *} P \leq 0.001\right)$.

of our screening strategy. The whole library included 14 microtubule-targeting drugs (MTDs), and all were active in our assay (Figure $3 \mathrm{C}$ and Supplemental Table 2). Strikingly, 2-methoxyestradiol (2-ME2) (36) exhibits structural differences from other MTDs suggesting distinctive properties (Figure 3C). Indeed, 2-ME2 is the only MTD for which we observed a differential response in AML cells (Supplemental Figure 3). Finally, MTDs have well-known toxicities toward HSPCs (37). When tested against purified human $\mathrm{Lin}^{-} \mathrm{CD} 34^{+}$cells from normal cord blood (38), all MTDs except 2-ME2 totally inhibited cell viability (Figure 3D). Interestingly,
2-ME2 did not affect the numbers of $\operatorname{Lin}^{-} \mathrm{CD} 34^{+}$cells compared with vehicle-treated control cultures (Figure 3D).

2-ME2 is an estrogen derivative that does not bind the estrogen receptor (ER) (36), and estrogens impaired pre-LSCs at micromolar concentrations only, well outside their bioactive range (Supplemental Figure 4A), indicating an ER-independent mechanism. Rather, 2-ME2 is an MTD (36). Dose-response curves of 2-ME2 on $S C L^{\text {tg }} L M O 1^{\text {tg }}$ pre-LSCs and normal DN3 thymocytes revealed $\mathrm{IC}_{50}$ of $570 \mathrm{nM}$ and $2 \mu \mathrm{M}$, respectively (Figure $3 \mathrm{E})$, suggesting that the $S C L-L M O 1$ oncogenes sensitize DN3 
thymocytes to 2-ME2. Furthermore, we observed that the nontoxic doses of 2-ME2 did not affect the capacity of WT DN3 thymocytes to generate DP cells in vitro (Supplemental Figure 4B), indicating that 2-ME2 did not block normal T cell differentiation within the tested range. In addition, we excluded the possibility that decreased pre-LSC viability could be secondary to stromal cell failure, since MS5-DL4 cell viability as well as DL4 surface expression was not affected by 2-ME2 at concentrations as high as $10 \mu \mathrm{M}$ (Figure 3F).

2-ME2 exhibits structural similarity to DEXA (Figure 3C). We therefore compared their activities on the self-renewal of pre-LSCs following in vitro treatments with $1 \mu \mathrm{M} 2-\mathrm{ME} 2$ or $5 \mathrm{nM}$ DEXA (Supplemental Figure 5A), corresponding to 75\% inhibition in vitro (Figure 3E and Figure 1B). Pre-LSC frequency and activity were quantitatively assessed in vivo, by transplantation in irradiated congenic mice in limiting dilution assays (Supplemental Figure 5A). As shown in Figure 3G (and Supplemental Figure 5B), there was a striking difference between DEXA and 2-ME2. Even though both compounds were used at a dose of $75 \%$ inhibition in vitro, $S C L^{t g} L M O 1^{t g}$ pre-LSCs fully recovered from DEXA treatment in culture and their self-renewal activity in vivo was not altered, concurring with the view that pre-LSCs are spared by current treatment $(4,24)$. In contrast, 2-ME2 reduced pre-LSC frequency 10 -fold (Figure 3G and Supplemental Figure 5) and is therefore a drug candidate for T-ALL treatment.

2-ME2 inhibits SCL accumulation and transcriptional activity. At the molecular level, 2-ME2 but not DEXA decreased the expression of the SCL-dependent self-renewal genes (14) Lyl1 and Hhex without affecting mRNA levels of SCL (Figure 4A). Since pre-LSC self-renewal activity depends on the capacity of SCL to activate transcription (14), we next addressed the question of whether 2-ME2 inhibits SCL transcription activity, using a quantitative reporter assay $(39,40)$. We observed that 2-ME2 but not DEXA inhibited SCL activity in transient transcription assays (Figure $4 \mathrm{~B}$ ), consistent with the suppression of endogenous SCL target genes in pre-LSCs (Figure 4A). These results concur with our functional studies (Figure $3 \mathrm{G}$ ) and indicated that 2-ME2 inhibits pre-LSC self-renewal activity via inhibition of SCL.

Recent evidence suggests an important nonmitotic function of 2-ME2, in preventing the nuclear accumulation of transcription factors or DNA repair proteins $(41,42)$. We therefore quantitated SCL at the single-cell level by flow cytometry and found that 2-ME2 caused a dose-dependent inhibition of the human SCL oncoprotein in $S C L^{\text {tg }} \mathrm{LMO1}{ }^{\text {tg }}$ pre-LSCs (Figure $4 \mathrm{C}$ ). We examined the fate of the SCL protein by live cell imaging throughout the time exposure to 2-ME2. To this end, we delivered SCL-GFP into HeLa cells. We then followed GFP expression in individual cells in the presence or absence of 2-ME2 for 16 hours, a time point chosen based on HeLa full viability in drug-treated cultures (Supplemental Figure 6A). SCL-GFP expression in the nucleus of 2-ME2treated cells decreased steadily compared with that in untreated cells (Figure 4, D and E, top panels, and Supplemental Figure 6B). In contrast, 2-ME2 treatment of HeLa cells stably expressing the nuclear protein histone H2B-RFP did not modify the level of RFP expression during the same time lapse (Figure 4, D and E, bottom panels, and Supplemental Figure 6C), showing that 2-ME2 specifically targets SCL accumulation.
Similarly, we found that 2-ME2 decreased endogenous SCL protein levels in the $\mathrm{SCL}^{+}$JURKAT T-ALL cell line, starting at 4-6 hours after treatment (Figure $4 \mathrm{~F}$ ), exactly as observed with transfected SCL in HeLa cells (Figure 4, D and E). Finally, our immunofluorescence approach confirmed that 2-ME2 decreases SCL without modifying histone $\mathrm{H} 3$ nuclear expression levels (Figure 4, G and H). 2-ME2 is a microtubule depolymerizing agent (43). To assess the possibility that decreased SCL protein levels could be secondary to the perturbation of microtubule dynamics, we treated JURKAT $\mathrm{T}$ cells with colchicine, a well-characterized microtubule depolymerizing drug. While colchicine also inhibited cell growth (Supplemental Figure 7A), this occurred in the absence of a noticeable effect on SCL protein levels (Supplemental Figure 7B). Together, these results demonstrate that 2-ME2 impairs SCL expression via a colchicine-independent mechanism.

2-ME2 targets 2 pathways: inhibition of SCL and MYC. Resistance to glucocorticoids is an adverse prognostic factor in T-ALL both at presentation and in relapse (44). We found that 2-ME2 induced apoptosis (Supplemental Figure 8A, right panel, and Supplemental Figure $8 \mathrm{~B}$ ) and efficiently inhibited the viability of human T-ALL cell lines (Figure 5A, right panel) that are either corticosensitive (P12-ICHIKAWA) or corticoresistant (JURKAT, KOPTK1, and HPB-ALL; Figure 5A, left panel, and Supplemental Figure 8A, left panel). Furthermore, 2-ME2 did not modify the corticoresistance of JURKAT cells (Supplemental Figure 8C, left panel) nor the corticosensitivity of P12-ICHIKAWA cells (Supplemental Figure 8C, right panel) or of SCL-LMO1 pre-LSCs (Supplemental Figure 8D), indicating that the activity of 2-ME2 is cortico-independent.

Consistent with the reported dependence of JURKAT cell viability on SCL (45), we observed that SCL protein expression in JURKAT cells (Figure 5B) was inhibited by 2-ME2 in a dosedependent manner (Figure $5 \mathrm{C}$ ) in exactly the same range as cell growth inhibition $(\sim 500 \mathrm{nM})$. This was associated with the downregulation of SCL target gene NKX3.1 (ref. 45 and Supplemental Figure 8E, left panel), while $S C L$ mRNA levels were not modified by 2-ME2, as observed in pre-LSCs.

We previously showed that the self-renewal node in pre-LSCs depends not only on the SCL oncogene but also on the NOTCH1/ $M Y C$ axis (14), a non-cell-autonomous pathway triggered by Deltalike NOTCH1 ligand. Importantly, 2-ME2 did not affect NOTCH1 target genes including Myc in pre-LSCs (Supplemental Figure 9A), ruling out the possibility that 2-ME2 could affect a general mechanism of NOTCH1 activation triggered by ligand endocytosis (46). In contrast, 2-ME2 decreased MYC protein levels in pre-LSCs (Supplemental Figure 9B). Interestingly, all tested T-ALL cell lines exhibit elevated MYC protein levels (Figure 5B and Supplemental Figure 9C), which were nearly abrogated by 2-ME2 (Figure 5, C and D), whereas MYC mRNA levels were unaffected (Supplemental Figure 8E, right panel). Strikingly, this inhibitory activity was not observed with 2 other factors controlling the self-renewal node in normal HSCs, HOXA9 and HOXB4, or with the SCL protein partner E2A (Figure 5E). Together, these results indicate a selective and previously unreported inhibitory effect of 2-ME2 on SCL and MYC protein levels.

Decreased SCL and MYC protein levels were not due to protein degradation, since 2-ME2 did not modify SCL and MYC halflives $\left(t_{1 / 2}\right)$, as determined by cycloheximide chase in all $3 \mathrm{~T}$-ALL 
cell lines (Figure 5F and Supplemental Figure 9D). We therefore examined the possibility that 2-ME2 affects $S C L$ and MYC translation. First we observed that the p70 ribosomal S6 kinase (S6K), a key downstream effector of mTORC1 signaling pathway (47), was active in all T-ALL cell lines (Figure 5G). Interestingly, 2-ME2 inhibits $\mathrm{S} 6$ phosphorylation in KOPTK1 and P12-ICHIKAWA and to a lesser extent in JURKAT cells (Figure 5G), indicating that 2-ME2 inhibits the mTORC1 pathway. Indeed, the translation of MYC mRNA was previously shown to be highly mTORC1-sensitive because of a long and structured 5'-UTR (48). By metabolic labeling with ${ }^{35} \mathrm{~S}$-methionine (Figure $5 \mathrm{H}$ and Supplemental Figure 9E), we found that 2-ME2 strongly decreased MYC translation (Figure $5 \mathrm{H}$, right panel). These results reveal an unsuspected mechanism of 2-ME2 in inhibiting MYC translation. Since SCL translation was not significantly affected by 2-ME2 (Figure $5 \mathrm{H}$, left panel), we infer that MYC and SCL translation has differential dependence on mTORC1 signaling.

2-ME2 kills leukemic blasts without affecting viability of normal HSPCs. We next showed that 2-ME2 inhibits SCL and MYC expression in fully transformed $S C L^{t g} L M O 1^{t g}$ leukemic blasts (Figure 5I) and addressed the question of whether 2-ME2 might also inhibit primary $S C L^{\text {tg }} L M O 1^{\text {tg }}$ leukemic cells. Comparison of the transcriptomes of murine SCL-LMO1-induced T-ALLs and of their normal counterparts points to the regulation of apoptosis as a top gene ontology category among SCL targets (Supplemental Figure 10A). We observed that 2-ME2 induced apoptosis in vitro both in pre-LSCs and in T-ALL blasts from $S C L^{\text {tg }} L M O 1^{\text {tg }}$ mice (Supplemental Figure 10B), consistent with an inhibition of SCL antiapoptotic function (49-51).

To study the effect of 2-ME2 on T-ALL in vivo, primary $S C L^{t g}$ $L M O 1^{t g} \mathrm{~T}-\mathrm{ALL}$ blasts were transplanted into 10 syngeneic mice (Figure 6A). After engraftment (Supplemental Figure 10C), which was as rapid and homogenous as in Figure 1E, mice were treated with 2-ME2 via implantation of osmotic minipumps $(40 \mathrm{mg} / \mathrm{kg} / \mathrm{d})$ (Figure 6A). Compared with control mice receiving the vehicle only, 2-ME2 induced a profound reduction of leukemic blasts in the BM, thymus, spleen, and peripheral blood (Figure 6, B and C) and altered their capacities to initiate leukemia in secondary recipients (Figure 6D). In contrast, the analysis of normal host (CD45.1 $1^{+}$T cell compartments indicated that 2-ME2 did not block normal $\mathrm{T}$ cell differentiation in vivo (Supplemental Figure 11A), confirming our in vitro results (Supplemental Figure 4B). Despite high MYC levels in normal DN3 thymocytes, 2-ME2 did not affect MYC expression levels in these cells (Supplemental Figure 11B), concurring with their low sensitivities to 2-ME2 (Figure 3E). We therefore conclude that the SCL and LMO1 oncogenes sensitize DN3 cells to 2-ME2.

In parallel, we monitored how 2-ME2 affects residual normal stem/progenitor cells in the same mice. Host HSCs can be distinguished from transplanted cells on the basis of CD45 isoforms, as shown in Figure 6A. 2-ME2 modifies neither the frequency (Figure 6B) nor the absolute numbers (Figure 6E) of host HSPCs as defined by their $\mathrm{c}-\mathrm{Kit}^{+} \mathrm{Sca}-1^{+}$lineage $\mathrm{e}^{-}$(KSL) phenotype in vivo, contrasting with its dramatic effect on transplanted leukemic cells in the same mice. Moreover, 2-ME2-treated KSL cells retained proliferative and multilineage differentiation potentials when transplanted in secondary recipients (Figure 6F), indicating that
2-ME2-treated HSPCs remain functional. Finally, in vitro treatment with 2-ME2 for 4 days was sufficient to alter pre-LSC activity (Figure 3G), while normal HSPCs (KSL) retained self-renewal activity (Figure 6G) and multipotency (Supplemental Figure 11C) in serial transplantation assays. Therefore, this preclinical model of T-ALL indicates that normal HSPCs remain functional after in vivo exposure to 2-ME2, concurring with the reported lack of myelosuppression in clinical trials (36). Similarly, normal $\mathrm{T}$ cells were at least 4-fold less sensitive to 2-ME2, corresponding to an inability to downregulate MYC. In contrast, 2-ME2 reduced T-ALL blasts, leukemia-propagating cells, and pre-LSCs, revealing a significant difference in chemosensitivity between normal cells and preleukemic/leukemic cells.

Primary human T-ALLs are sensitive to 2-ME2. Xenograft models of human T-ALL mimic disease relapse (52) and represent a powerful preclinical tool to directly test drug efficacy on primary leukemia cells in vivo (28). Purified leukemic blasts from 2 T-ALL patients (14H025 and 14H148; Supplemental Table 3) were transplanted and expanded into immunocompromised NOD.CgPrkd ${ }^{\text {scid } I l 2 r g^{t m W j l} / S z J}$ (referred to here as NSG) mice (Figure 7A) to test their sensitivities to 2-ME2 both in vitro and in vivo.

In vitro, both patient samples were sensitive to 2-ME2 treatment in a dose-dependent manner but resistant to DEXA (Figure 7B). At the molecular level, patient 14H025 exhibited SIL-TAL1 chromosomal rearrangement and patient $14 \mathrm{H} 148$ did not (Supplemental Figure 12A and Supplemental Table 4). 2-ME2 treatment efficiently downregulated SCL protein levels in the SIL-TAL1 patient sample 14H025 (Figure 7C), associated with the inhibition of 2 SCL-dependent target genes, LYL1 and NKX3.1 (45) (Figure $7 \mathrm{D}$, left panel). In contrast, $L Y L 1$ remained unaffected in the other patient sample, while NKX3.1 was decreased via an SCL-independent mechanism (Figure 7D, right panel). MYC protein expression was detectable in both patient samples despite the absence of NOTCH1 mutations and of MYC rearrangement (Supplemental Table 4). Interestingly, MYC was decreased by 2-ME2 treatment in vitro in both patient samples (Figure 7C), associated with the inhibition of the well-established MYC target gene ODC (reviewed in ref. 53) (Figure 7D). These observations indicate that 2-ME2 inhibits SCL and MYC functions in primary human T-ALL samples, as observed in the mouse model.

Finally, mice were transplanted and monitored for engraftment before treatment with 2-ME2 using osmotic minipumps (Figure 7E). Within 7 days, both samples engrafted in the BM with dissemination in the spleen, and 14H148 engrafted in the peripheral blood as well (Supplemental Figure 12B). Strikingly, treatment with 2-ME2 downregulated SCL and MYC protein levels in vivo (Supplemental Figure 12C) and prevented T-ALL progression by significantly reducing the leukemic burden in the $\mathrm{BM}$, spleen (Figure $7 \mathrm{~F}$ ), and peripheral blood (Supplemental Figure 12B) of recipient mice.

Together, these results indicate that 2-ME2 exhibits antileukemic activity in primary human T-ALL blasts.

\section{Discussion}

In the present study, we bring evidence that pre-LSCs are more chemoresistant than leukemic blasts to conventional therapies because of their low proliferation rate. We developed a robust heterotypic assay for chemical screening of primary pre-LSCs in 
an ex vivo assay mimicking a thymic-like microenvironment, and demonstrated the feasibility of this strategy for HTS. This strategy led us to identify an inhibitor that targets 2 central actors of the self-renewal node in pre-LSCs, SCL and MYC (14).

The development of new drugs targeting self-renewing (pre) cancer stem cells could open new therapeutic opportunities for more selective treatments (6). In T-ALL, leukemic stem cells are capable of sustained leukemia propagation. Nonetheless, these cells are endowed with an evolving genetic landscape, carrying founder mutations as well as varying secondary mutations (24, 52). In contrast, the emergence of self-renewing pre-LSCs via oncogene-induced reprogramming of normal thymocytes represents the earliest events in T-ALL $(10,12,14)$ and in B cell acute lymphoblastic leukemia (7). Therefore, pre-LSCs represent the prime cellular target for novel drug development. In AML, these cells are believed to harbor landscaping mutations that remain stable in remission and relapse (4). These molecular studies also suggested that the cells that caused relapse are preleukemic HSCs that do not have the full complement of transforming events. Obviously, these cells must have escaped therapy (4). Nonetheless, direct evidence for differential chemosensitivity between pre-LSCs and leukemic blasts was hindered by the extreme rarity of these cells within the tumor bulk, as we now show that pre-LSCs are rapidly offset by more competitive, fully transformed leukemic blasts. We took advantage of the mouse model that recapitulates the evolution of the human disease to demonstrate that pre-LSCs are less sensitive than leukemic blasts to drugs that target proliferating cells, such as topoisomerase inhibitors, because of their low proliferative status. Similarly, leukemic blasts and pre-LSCs also exhibit differential sensitivities to radiation (54).

Our previous work indicates that self-renewing pre-LSCs depend both on the primary oncogenes and on the physiological NOTCH1 pathway activated by the microenvironment (14). Interestingly, 2-ME2 specifically prevents SCL accumulation via a colchicine-independent mechanism, which differs from the colchicine-sensitive transport of HIF- $1 \alpha$ into the nucleus via microtubules (36). Moreover, 2-ME2 also decreased MYC protein levels, resulting in decreased expression of a prototypic MYC target gene, $O D C$. Based on our previous finding that $M Y C$ can substitute for NOTCH1 signaling to collaborate with $S C L-L M O 1$ in thymocyte reprogramming (14), we conclude that 2-ME2 inhibits pre-LSCs by antagonizing their dependence on both $S C L$ and the DL4NOTCH1 pathway. Moreover, we showed that 2-ME2 inhibits SCL and MYC targets in primary human T-ALL blasts and prevents their growth in a preclinical model of T-ALL.

Interestingly, gain of function of NOTCH1 is the most frequent genetic alteration in T-ALL that can be found in all molecular subgroups, defined by $S C L / T A L 1, L M O 2, T L X 1 / 3, H O X A$, and $M Y B$ (9, $26,55)$. Given the necessary collaboration of NOTCH1-MYC with $S C L-L M O 1$ in self-renewing pre-LSCs (14) and the importance of NOTCH1-MYC in T-ALL $(56,57)$, we surmise that inhibiting MYC could be lethal in other molecular T-ALL subgroups. In addition, inhibition of the NOTCH signaling pathway can antagonize glucocorticoid resistance, an adverse prognostic factor in ALL (58). We propose that 2-ME2 will be beneficial for the treatment of glucocorticoid-resistant primary or relapsed human T-ALL. In addition, 2-ME2 may be of interest in AML treatment as well because of its inhibitory effect on MYC. Concurring with the emerging role of MYC in AML $(59,60)$, perphenazine, the only phenothiazine hit in $\mathrm{AML}$ in the present study, was revealed as a top hit in a chemical screen for suppressors of MYC-induced T-ALL in zebrafish (35). Finally, the multiparametric screening assay described here could potentially allow for the identification of compounds that restore cell differentiation in T-ALL, as exemplified by the differentiation therapy achieved in acute promyelocytic leukemia (61).

The importance of regulated translation in T-ALL is underscored by the finding of recurring loss-of-function mutations in several ribosomal genes, RPL5, RPL10, and RPL22, in 10\%-20\% of pediatric T-ALL (62). In addition, PTEN deletion occurs in $20 \%$ of T-ALL, causing increased AKT (63) and a hyperactive mTORC1 pathway. The translation of mRNA into polypeptide is mostly controlled at the level of translation initiation, which is partly regulated by the mTORC1 signaling pathway (47). While mTORC1 controls global protein synthesis, it preferentially stimulates the translation of selected groups of mRNAs that contain relatively long and structured 5'-UTRs, including MYC (47). Here, we show that 2-ME2 strongly alters $M Y C$ translation without modifying $S C L$ translation. We thus conclude that MYC and SCL have differential dependence on the mTORC1 pathway. It remains to be determined whether this differential dependence could be imparted by the structure of their 5'-UTRs, as reported for MYC (47). In summary, our observations place MYC as a highly sensitive target hit of 2-ME2, downstream of the mTORC1 pathway. Finally, MYC is frequently dysregulated in tumors and represents a major target in cancer therapy (64). In addition to inhibiting MYC transcription or activity (65), our observations support the view that it is possible to target MYC translation.

SCL controls HSC self-renewal activity under stress (50, 6668), and is redundant with $L y l 1$ during steady-state hematopoiesis (69). In contrast, $S C L$ activation in thymocytes occurs in a context whereby other self-renewal genes are absent and therefore preLSCs are "addicted" to SCL. Along the same line, Lyl1 upregulation by $\mathrm{Lmo} 2$ in DN3 thymocytes occurs in a context whereby $\mathrm{Scl}$ is not expressed, and therefore $L y l 1$ is required for $L m o 2$ reprogramming activity (70). Similarly, $M y c$ is critical for pre-LSC selfrenewal activity $(14,56)$, whereas $M y c$ is redundant in HSCs $(71)$, indicating that targeting MYC will spare normal HSCs. Our results do not rule out the possibility that normal stem/progenitor cells express a 2-ME2-resistant isoform of tubulin, $\beta_{1}$-tubulin, proposed to protect patients from drug-induced myelosuppression (72). Nonetheless, we favor the idea that differential dependence on SCL and MYC levels distinguishes pre-LSCs from normal HSCs. Hence, decreasing SCL and MYC protein levels is synthetic lethal in pre-LSCs but not in HSCs that are endowed with a highly redundant self-renewal node.

Chemical biology combined with the capacity for HTS provides unprecedented opportunities for drug discovery. Yet, despite the number of hits, there is currently a tendency for high drug attrition rates when it comes to efficacy in clinical trials (73). Without ruling out the possibility that many factors could contribute to this high attrition rate, our results suggest that cell line-based screening as well as 2-dimensional screening assays may not yield muchneeded novel bioactive compounds. While these assays have been useful in identifying anticancer drugs, it is well known that cell 
fate is shaped by the local niche. It is therefore not surprising that the niche may also modify the chemosensitivity of primary leukemic blasts (74). Our strategy led to the identification of a drug that inhibits both cell-autonomous and non-cell-autonomous pathways, acting in vitro and in vivo, while sparing normal HSCs. We propose that recapitulating tissue-like conditions (75-77) represents a promising avenue in the quest for new cancer therapies in other tumors.

\section{Methods}

Supplemental Methods are available online with this article; doi:10.1172/JCI86489DS1.

Human leukemia samples and cell lines. All AML and T-ALL samples were collected from the Maisonneuve-Rosemont Hospital of Montreal, Canada. Human JURKAT, KOPTK1, P12-ICHIKAWA, and HPBALL cell lines were obtained from DSMZ, Germany.

Mice and ethics statement. All animals were maintained in pathogen-free conditions. The current study was performed using previously described SIL-SCL (referred to as $S C L^{t g}$ ) and Lck-LMO1 (referred to as $\left.L M O 1^{t g}\right)$ mice $(15,16,33)$. All mouse lines were backcrossed onto a C57BL6/J background for more than 12 generations. Mice cohorts were generated by cross-breeding, and their genotypes were verified by PCR.

Generation of MS5-DL4 stromal cell lines. Mouse stromal MS5 cells than can maintain in vitro both mouse and human long-term hematopoiesis were from K. Mori (Nagata University, Japan) (78) and were maintained as described previously (30). VSV-G-producing cells transfected with the human DLL4 cDNA (MHS4426-98361330, GE Healthcare) were used for gene transduction in MS5 cells (48 hours) as described previously $(50,66)$. Transduced MS5 cells were stained using rabbit anti-DLL4 (ab7280, Abcam), and 3 MS5 stromal cell lines were generated by cell sorting according to 3 levels of DL4 expression (low, medium, and high). Staining with the secondary antibody only was used for negative controls.

Chemical screen of small molecules on pre-LSCs. MS5-DL4 stromal cells $\left(2 \times 10^{3}\right.$ per $40 \mu \mathrm{l}$ per well) were seeded in 384 -well plates in complete $\mathrm{T}$ cell medium described in the Supplemental Methods. Twenty-four hours later, DN thymocytes from thymi of 3-week-old preleukemic $S C L^{\text {tg }} L M O 1^{\text {tg }}$ mice were prepared by depletion of $\mathrm{CD} 4^{+}$ and $\mathrm{CD} 8^{+}$thymocytes through immunomagnetic bead cell separation as previously described (66). DN3 (pre-LSC) thymocytes $\left(5 \times 10^{3}\right.$ per $5 \mu \mathrm{l}$ per well) were then purified and cocultured on seeded MS5-DL4 stromal cells by cell sorting. Twenty-four hours later, cocultured preLSCs were exposed to 1,904 individual FDA-approved and drug-like small molecules (Preswick; Biomol; Lopac/Sigma-Aldrich; MicroSource Discovery Systems) at a dose of $500 \mathrm{nM}$. Absolute numbers of DN3 cells were determined after 5 days of culture using flow cytometry. In each plate, 16 wells containing pre-LSCs were treated with 1 $\mu \mathrm{M} \gamma$-secretase inhibitor (GSI) and 16 wells were treated with DMSO (vehicle) as positive and negative controls, respectively (Supplemental Figure 2). The inhibition (percentage) of pre-LSC viability by individual compounds was calculated based on the absolute numbers of cells in the DN3 gate (Thy $\left.1^{+} \mathrm{CD} 4^{-} \mathrm{CD} 8^{-} \mathrm{CD} 25^{+} \mathrm{CD} 44^{-}\right)$recovered from 3 different treatment conditions according to the formula 100 - ((test compound - median GSI) / (median vehicle - median GSI) $\times 100$ ).

Pre-LSCs were stained by addition of $5 \mu \mathrm{l} /$ well of PBS supplemented with $2 \%$ FBS containing PE-anti-Thy1.2, PE-Cy5-anti-CD4,
APC-anti-CD8, PE-Cy7-anti-CD25, and AF780-anti-CD44 at $4^{\circ} \mathrm{C}$ for 15 minutes. The screen was then performed with an LSRII flow cytometer equipped with an HTS module (BD Biosciences).

FACS analysis and cell sorting. Immunostaining was done as previously described (33). Dead cells were excluded by propidium iodide staining. Immunophenotype and apoptosis analysis was performed on an LSRII cytometer using DIVA (BD Biosciences) software. Cell sorting was done using an FACSAria cytometer (BD Biosciences).

Congenic transplantation assay. Preleukemic thymocytes from donor mice $\left(\mathrm{CD} 45.2^{+}\right)$were transplanted in i.v. into sublethally irradiated (600 cGy) recipient mice (CD45.1 ) (14). Thymic chimerism in the $\mathrm{T}$ lineage (Thy1.2+) was analyzed by flow cytometry (FACS) and illustrated by the percentage of donor-derived cells (\% CD45.2+) found in the recipient thymus.

In vivo study of the effect of 2-ME2 on T-ALL development and HSC functions. T-ALL blasts (CD45.2 $)$ from primary $S C L^{\text {tg }} L M O 1^{\text {tg }}$ thymoma were injected i.v. into 10 sublethally irradiated CD $45.1^{+}$recipient mice $\left(5 \times 10^{6}\right.$ blasts per mouse). T-ALL reconstitution was assessed by FACS after BM aspiration, and mice were randomly selected for treatment with vehicle alone (50\% DMSO; 50\% PEG400) or 2-ME2 (Bosche Scientific, M19071; 40 mg/kg/d). Osmotic minipumps (Alzet model 2001; $1 \mu \mathrm{l} / \mathrm{h}, 7$ days) containing vehicle or 2-ME2 were loaded and primed at $37^{\circ} \mathrm{C}$ in $0.9 \% \mathrm{NaCl}, 24$ hours before their subcutaneous implantation, in accordance with the manufacturer's instructions. T-ALL reconstitution was monitored in the thymus, BM, spleen, and blood, and engrafted T-ALL blasts from the BM were transplanted into secondary sublethally irradiated $\mathrm{CD} 45.1^{+}$mice. c-Kit ${ }^{+} \mathrm{Sca}-1^{+}$lineage ${ }^{-}$ (KSL) cells from the BM of treated CD $45.1^{+}$mice were transplanted into secondary lethally irradiated CD $45.2^{+}$mice in parallel.

Xenogenic transplantation assay. T-ALL patient samples were selected for high blast counts (>90\%) in the peripheral blood. T-ALL blasts were immunophenotyped using FITC-anti-hCD45, PE-antihCD7, PE-Cy7-anti-hCD4, PE-Cy5-anti-hCD8, and APC-antihCD34. Normal peripheral $\mathrm{CD} 34^{+} \mathrm{CD} 7^{-}$hematopoietic progenitors and mature $\mathrm{CD}^{+/ / h i}$ and $\mathrm{CD}^{+/ \text {hi }}$ single-positive $\mathrm{T}$ cells (28) were excluded by cell sorting, and T-ALL blasts were transplanted and expanded by transplantation into sublethally irradiated (250 cGy) 8to 12-week-old NOD-Scid IL2rg ${ }^{\text {null }}$ mice (NSG, Jackson Laboratory). Engrafted T-ALL blasts were collected from the spleen and the BM 6-8 weeks after transplantation.

T-ALL blasts $\left(\mathrm{CD} 45^{+} \mathrm{CD}^{+}\right)$from primary NSG mice engrafted with each patient sample were injected i.v. into 10 secondary NSG mice $(5 \times$ $10^{6}$ blasts per mouse). T-ALL reconstitution was assessed by FACS after $\mathrm{BM}$ aspiration, and mice were randomly selected for treatment with the vehicle or 2-ME2 using osmotic minipumps as described above.

Limiting dilution assays. Preleukemic thymocytes were transplanted into irradiated hosts at various cell doses. Mice were scored positive when $\mathrm{T}$ cell lineage reconstitution was more than $0.1 \%$ in the thymus. Pre-LSCs frequencies (range pre-LSC $\pm \mathrm{CI}$ ), also named competitive repopulating unit frequencies, were calculated by application of Poisson statistics using Limiting Dilution Analysis software (Stem Cell Technologies) (66).

Metabolic radiolabeling with ${ }^{35} S$-methionine and immunoprecipitation. $4 \times 10^{7}$ JURKAT cells $\left(10^{6}\right.$ cells $\left./ \mathrm{ml}\right)$ were incubated overnight at $37^{\circ} \mathrm{C}$, washed twice with $50 \mathrm{ml}$ of L-methionine-free DMEM (Wisent Inc., 319-045-CL), and pretreated or not (DMSO) with 2-ME2 $(3 \mu \mathrm{M})$ for 30 minutes. Cells were then centrifuged to 
remove the supernatant, resuspended in fresh L-methionine-free DMEM containing $1.75 \mathrm{mCi}$ of ${ }^{35} \mathrm{~S}$-methionine (PerkinElmer), and incubated for 3 hours in the presence or absence (DMSO) of 2-ME2 $(3 \mu \mathrm{M})$. JURKAT cells were washed 3 times with ice-cold PBS and harvested in $600 \mu \mathrm{l}$ lysis buffer $\left(10 \mathrm{mM} \mathrm{K}_{3} \mathrm{PO}_{4}, 1 \mathrm{mM}\right.$ EDTA, $5 \mathrm{mM}$ EGTA, $10 \mathrm{mM} \mathrm{MgCl}$, $50 \mathrm{mM} \beta$-glycerophosphate, $0.5 \%$ Nonidet P-40, 0.1\% Brij 35, 0.1\% deoxycholic acid, $1 \mathrm{mM}$ sodium orthovanadate $\left[\mathrm{Na}_{3} \mathrm{VO}_{4}\right], 1 \mathrm{mM}$ PMSF, and a Complete Protease Inhibitor Mixture tablet [Roche]). For immunoprecipitations, cell lysates were incubated with the SCL/TAL1 (C-21) sc-12984 goat polyclonal antibody or with the c-MYC (9E10) sc-40 mouse monoclonal antibody for 2 hours, followed by a 1-hour incubation with protein ASepharose CL-4B beads (GE Healthcare). Immunoglobulins from goat (Sigma-Aldrich, I-9140) and mouse (Sigma-Aldrich, I-8765) serum were used as control for the immunoprecipitation. Immunoprecipitates were washed 3 times in lysis buffer, and beads were eluted and boiled in $2 \times$ reducing sample buffer $(125 \mathrm{mM}$ Tris- $\mathrm{HCl}$ [pH 6.8], 20\% [vol/vol] glycerol, 4\% [wt/vol] SDS, 6.2\% DTT, and $0.1 \%$ bromophenol blue). Immunoblotting was performed using a submersible transfer apparatus and polyvinylidene fluoride membrane. Radiolabeling was revealed using a PhosphorImager (GE Healthcare Life Science).

Statistics. Z' value was calculated according to the formula $1-[3 \times(S D$ of positive control $+\mathrm{SD}$ of negative control)/(mean of positive control mean of negative control)]. The theoretical maximum value for the $Z^{\prime}$ factor is 1 , and ideally the $Z^{\prime}$ factor is between 0.5 and 1 .

We performed statistical analyses by Student's $t$ test $\left({ }^{*} P \leq 0.05\right.$, $\left.{ }^{* *} P \leq 0.01,{ }^{* * *} P \leq 0.001\right)$. We assumed normality and equal distribution of variance between the different groups analyzed. Survival in mouse experiments was represented with Kaplan-Meier curves, and significance was estimated with log-rank test (GraphPad Prism).

Study approval. All AML and T-ALL samples were collected with informed consent according to procedures of the Banque de Cellules Leucémiques du Québec, and the project was approved by the Research Ethics Boards of Maisonneuve-Rosemont Hospital and the University of Montreal. All animals were maintained in pathogen-free conditions according to institutional animal care and guidelines set by the Canadian Council on Animal Care.

\section{Author contributions}

BG, PSM, BHK, and TH designed the study. BG, DFTV, SN, IF, JO, GL, JR, JK, MT, EO, MK, VL, AH, and DG performed experiments. BG, DFTV, PSM, AM, PPR, BHK, and TH analyzed data. JH, JC and GS contributed to experiments with primary human cells. BG and TH interpreted the data and wrote the manuscript. All authors contributed to the final draft.

\section{Acknowledgments}

We thank Danièle Mathieu (INSERM, Marseille, France) for the anti-SCL antibody, Adolfo Ferrando (Colombia University, New York, New York, USA) for the human T-ALL cell lines, Danièle Gagné and Gael Dulude for assistance with flow cytometry, Valérie Villeneuve for sequencing, and Jean Duchaine for HTS and for the chemical library. Clinical specimens were collected and analyzed by the Banque de Cellules Leucémiques du Québec, supported by the Cancer Research Network of the Fonds de Recherche du Québec en Santé (FRQS). The Institute for Research in Immunology and Cancer is supported in part by the Canada Foundation for Innovation and the FRQS. This work was funded by grants from the Canadian Institutes for Health Research (CIHR) and the Canadian Cancer Society Research Institute (to TH, PR, BK, and GS); the Leukemia Lymphoma Society of Canada, the Cancer Research Society Inc., and the Programme de soutien à la recherche (PSR), volet Soutien à des initiatives internationales de recherche et d'innovation (SIIRI), Ministère de l'Économie, de la Science et de l'Innovation-Québec (to TH); "Fonds de partenariat pour un Québec innovant et en santé" and the Institute for Research in Immunology and Cancer-Commercialization of Research (to TH); the Canada Research Chair program (to PR and GS); the Industrielle-Alliance research chair in leukemia at Université de Montréal (to JH); and fellowships from the Cole Foundation (to BG and DFTV), the CIHR (to EO), and the FRQS (to $\mathrm{BG})$. The funders had no role in study design, data collection and analysis, decision to publish, or preparation of the manuscript.

Address correspondence to: Trang Hoang, Institute for Research in Immunology and Cancer (IRIC), University of Montréal, PO Box 6128, Downtown Station, Montréal, Québec, Canada H3C 3J7. Phone: 514.343.6970; E-mail: trang.hoang@umontreal.ca.

DFTV's present address is: Jackson Laboratory for Genomic Medicine, Farmington, Connecticut, USA.

MT's present address is: McGill Cancer Center, Montreal, Quebec, Canada.

MK's present address is: Department of Pharmaceutical Sciences, Faculty of Pharmacy, University of Toronto, Toronto, Ontario, Canada.
1. Ishikawa F, et al. Chemotherapy-resistant human AML stem cells home to and engraft within the bone-marrow endosteal region. Nat Biotechnol. 2007;25(11):1315-1321.

2. McMillin DW, et al. Tumor cell-specific bioluminescence platform to identify stroma-induced changes to anticancer drug activity. Nat Med. 2010;16(4):483-489.

3. Lane SW, Scadden DT, Gilliland DG. The leukemic stem cell niche: current concepts and therapeutic opportunities. Blood. 2009;114(6):1150-1157.

4. Corces-Zimmerman MR, Hong WJ, Weissman IL, Medeiros BC, Majeti R. Preleukemic muta- tions in human acute myeloid leukemia affect epigenetic regulators and persist in remission. Proc Natl Acad Sci U S A. 2014;111(7):2548-2553.

5 . Shlush LI, et al. Identification of pre-leukaemic haematopoietic stem cells in acute leukaemia. Nature. 2014;506(7488):328-333.

6. Durinck K, et al. Novel biological insights in T-cell acute lymphoblastic leukemia. Exp Hematol. 2015;43(8):625-639.

7. Hong $\mathrm{D}$, et al. Initiating and cancer-propagating cells in TEL-AML1-associated childhood leukemia. Science. 2008;319(5861):336-339.

8. Ferrando AA, et al. Gene expression signatures define novel oncogenic pathways in T cell acute lymphoblastic leukemia. Cancer Cell. 2002;1(1):75-87.

9. Van Vlierberghe P, Pieters R, Beverloo HB, Meijerink JP. Molecular-genetic insights in paediatric T-cell acute lymphoblastic leukaemia. Br J Haematol. 2008;143(2):153-168.

10. Hoang T. Of mice and men: how an oncogene transgresses the limits and predisposes to $\mathrm{T}$ cell acute lymphoblastic leukemia. Sci Transl Med. 2010;2(21):21ps10.

11. Krivtsov AV, et al. Transformation from committed progenitor to leukaemia stem cell initiated by 
MLL-AF9. Nature. 2006;442(7104):818-822.

12. McCormack MP, et al. The Lmo2 oncogene initiates leukemia in mice by inducing thymocyte self-renewal. Science. 2010;327(5967):879-883.

13. Cozzio A, Passegué E, Ayton PM, Karsunky H, Cleary ML, Weissman IL. Similar MLL-associated leukemias arising from self-renewing stem cells and short-lived myeloid progenitors. Genes Dev. 2003;17(24):3029-3035.

14. Gerby B, et al. SCL, LMO1 and Notch1 reprogram thymocytes into self-renewing cells. PLoS Genet. 2014;10(12):e1004768.

15. Aplan PD, et al. An scl gene product lacking the transactivation domain induces bony abnormalities and cooperates with LMO1 to generate T-cell malignancies in transgenic mice. $E M B O J$. 1997;16(9):2408-2419.

16. Tremblay M, et al. Modeling T-cell acute lymphoblastic leukemia induced by the SCL and LMO1 oncogenes. Genes Dev. 2010;24(11):1093-1105.

17. Weng AP, et al. Activating mutations of NOTCH1 in human $\mathrm{T}$ cell acute lymphoblastic leukemia. Science. 2004;306(5694):269-271.

18. Messersmith WA, et al. A Phase I, dose-finding study in patients with advanced solid malignancies of the oral $\gamma$-secretase inhibitor PF-03084014. Clin Cancer Res. 2015;21(1):60-67.

19. Krop I, et al. Phase I pharmacologic and pharmacodynamic study of the gamma secretase (Notch) inhibitor MK-0752 in adult patients with advanced solid tumors. JClin Oncol. 2012;30(19):2307-2313.

20. van Es JH, et al. Notch $/ \gamma$-secretase inhibition turns proliferative cells in intestinal crypts and adenomas into goblet cells. Nature. 2005;435(7044):959-963.

21. Weng AP, et al. c-Myc is an important direct target of Notch1 in T-cell acute lymphoblastic leukemia/ lymphoma. Genes Dev. 2006;20(15):2096-2109.

22. Palomero T, et al. NOTCH1 directly regulates c-MYC and activates a feed-forward-loop transcriptional network promoting leukemic cell growth. Proc Natl Acad Sci U S A. 2006;103(48):18261-18266.

23. Roderick JE, et al. c-Myc inhibition prevents leukemia initiation in mice and impairs the growth of relapsed and induction failure pediatric T-ALL cells. Blood. 2014;123(7):1040-1050.

24. Ding L, et al. Clonal evolution in relapsed acute myeloid leukaemia revealed by whole-genome sequencing. Nature. 2012;481(7382):506-510.

25. Pui CH, Carroll WL, Meshinchi S, Arceci RJ. Biology, risk stratification, and therapy of pediatric acute leukemias: an update. JClin Oncol. 2011;29(5):551-565

26. Aifantis I, Raetz E, Buonamici S. Molecular pathogenesis of T-cell leukaemia and lymphoma. Nat Rev Immunol. 2008;8(5):380-390.

27. Cleveland SM, et al. Lmo2 induces hematopoietic stem cell-like features in T-cell progenitor cells prior to leukemia. Stem Cells. 2013;31(5):882-894.

28. Gerby B, et al. Expression of CD34 and CD7 on human T-cell acute lymphoblastic leukemia discriminates functionally heterogeneous cell populations. Leukemia. 2011;25(8):1249-1258.

29. Schmitt TM, Zúñiga-Pflücker JC. Induction of $\mathrm{T}$ cell development from hematopoietic progenitor cells by delta-like-1 in vitro. Immunity.
2002;17(6):749-756.

30. Armstrong F, et al. NOTCH is a key regulator of human T-cell acute leukemia initiating cell activity. Blood. 2009;113(8):1730-1740.

31. Koch U, et al. $\Delta$-Like 4 is the essential, nonredundant ligand for Notch1 during thymic T cell lineage commitment. JExp Med. 2008;205(11):2515-2523.

32. Mohtashami M, Shah DK, Nakase H, Kianizad K, Petrie HT, Zúñiga-Pflücker JC. Direct comparison of Dll1- and Dll4-mediated Notch activation levels shows differential lymphomyeloid lineage commitment outcomes. Jimmunol. 2010;185(2):867-876.

33. Herblot S, Steff AM, Hugo P, Aplan PD, Hoang T. SCL and LMO1 alter thymocyte differentiation: inhibition of E2A-HEB function and pre-T $\alpha$ chain expression. Nat Immunol.2000;1(2):138-144.

34. Chervinsky DS, Zhao XF, Lam DH, Ellsworth M, Gross KW, Aplan PD. Disordered T-cell development and T-cell malignancies in SCL LMO1 double-transgenic mice: parallels with E2A-deficient mice. Mol Cell Biol. 1999;19(7):5025-5035.

35. Gutierrez A, et al. Phenothiazines induce PP2Amediated apoptosis in $\mathrm{T}$ cell acute lymphoblastic leukemia. JClin Invest. 2014;124(2):644-655.

36. Mueck AO, Seeger H. 2-Methoxyestradiolbiology and mechanism of action. Steroids. 2010;75(10):625-631.

37. McDermott SP, et al. A small molecule screening strategy with validation on human leukemia stem cells uncovers the therapeutic efficacy of kinetin riboside. Blood. 2012;119(5):1200-1207.

38. Fares I, et al. Cord blood expansion. Pyrimidoindole derivatives are agonists of human hematopoietic stem cell self-renewal. Science. 2014;345(6203):1509-1512.

39. Lécuyer E, et al. Protein stability and transcription factor complex assembly determined by the SCL-LMO2 interaction. J Biol Chem. 2007;282(46):33649-33658

40. Goardon N, et al. ETO2 coordinates cellular proliferation and differentiation during erythropoiesis. EMBO J. 2006;25(2):357-366.

41. Carbonaro M, O'Brate A, Giannakakou P. Microtubule disruption targets HIF-1alpha mRNA to cytoplasmic P-bodies for translational repression. J Cell Biol. 2011;192(1):83-99.

42. Poruchynsky MS, et al. Microtubule-targeting agents augment the toxicity of DNA-damaging agents by disrupting intracellular trafficking of DNA repair proteins. Proc Natl Acad Sci U S A. 2015;112(5):1571-1576

43. D’Amato RJ, Lin CM, Flynn E, Folkman J, Hamel E. 2-Methoxyestradiol, an endogenous mammalian metabolite, inhibits tubulin polymerization by interacting at the colchicine site. Proc Natl Acad Sci U S A. 1994;91(9):3964-3968.

44. Tissing WJ, Meijerink JP, den Boer ML, Pieters R. Molecular determinants of glucocorticoid sensitivity and resistance in acute lymphoblastic leukemia. Leukemia. 2003;17(1):17-25.

45. Kusy S, et al. NKX3.1 is a direct TAL1 target gene that mediates proliferation of TAL1-expressing human $\mathrm{T}$ cell acute lymphoblastic leukemia. JExp Med.2010;207(10):2141-2156.

46. Meloty-Kapella L, Shergill B, Kuon J, Botvinick E, Weinmaster G. Notch ligand endocytosis generates mechanical pulling force dependent on dynamin, epsins, and actin. Dev Cell. 2012;22(6):1299-1312.

47. Nandagopal N, Roux PP. Regulation of global and specific mRNA translation by the MTOR signaling pathway. Translation (Austin). 2015;3(1):e983402.

48. Wolfe AL, et al. RNA G-quadruplexes cause eIF4A-dependent oncogene translation in cancer. Nature. 2014;513(7516):65-70.

49. Palii CG, et al. Differential genomic targeting of the transcription factor TAL1 in alternate haematopoietic lineages. $E M B O ~ J .2011 ; 30(3): 494-509$.

50. Lacombe J, et al. Genetic interaction between Kit and Scl. Blood. 2013;122(7):1150-1161.

51. Sanda T, et al. Core transcriptional regulatory circuit controlled by the TAL1 complex in human T cell acute lymphoblastic leukemia. Cancer Cell. 2012;22(2):209-221.

52. Clappier E, et al. Clonal selection in xenografted human $\mathrm{T}$ cell acute lymphoblastic leukemia recapitulates gain of malignancy at relapse. JExp Med. 2011;208(4):653-661.

53. Gordan JD, Thompson CB, Simon MC. HIF and c-Myc: sibling rivals for control of cancer cell metabolism and proliferation. Cancer Cell. 2007;12(2):108-113.

54. Shields BJ, Alserihi R, Nasa C, Bogue C, Alexander WS, McCormack MP. Hhex regulates Kit to promote radioresistance of self-renewing thymocytes in Lmo2-transgenic mice. Leukemia. 2015;29(4):927-938

55. Tremblay CS, Hoang T, Hoang T. Early T cell differentiation lessons from T-cell acute lymphoblastic leukemia. Prog Mol Biol Transl Sci. 2010;92:121-156.

56. Herranz D, et al. A NOTCH1-driven MYC enhancer promotes $\mathrm{T}$ cell development, transformation and acute lymphoblastic leukemia. Nat Med. 2014;20(10):1130-1137.

57. Yashiro-Ohtani Y, et al. Long-range enhancer activity determines Myc sensitivity to Notch inhibitors in T cell leukemia. Proc Natl Acad Sci U S A. 2014;111(46):E4946-E4953.

58. Real PJ, et al. Gamma-secretase inhibitors reverse glucocorticoid resistance in $\mathrm{T}$ cell acute lymphoblastic leukemia. Nat Med. 2009;15(1):50-58.

59. Brondfield S, et al. Direct and indirect targeting of MYC to treat acute myeloid leukemia. Cancer Chemother Pharmacol. 2015;76(1):35-46.

60. Salvatori B, et al. Critical Role of c-Myc in Acute Myeloid Leukemia Involving Direct Regulation of miR-26a and Histone Methyltransferase EZH2. Genes Cancer. 2011;2(5):585-592.

61. Nasr R, et al. Eradication of acute promyelocytic leukemia-initiating cells through PML-RARA degradation. Nat Med.2008;14(12):1333-1342.

62. De Keersmaecker K, et al. Exome sequencing identifies mutation in CNOT3 and ribosomal genes RPL5 and RPL10 in T-cell acute lymphoblastic leukemia. Nat Genet. 2013;45(2):186-190.

63. Palomero T, et al. Mutational loss of PTEN induces resistance to NOTCH1 inhibition in T-cell leukemia. Nat Med. 2007;13(10):1203-1210.

64. Dang CV. MYC on the path to cancer. Cell. 2012;149(1):22-35.

65. Posternak V, Cole MD. Strategically targeting MYC in cancer. F1000Res. 2016;5. 
66. Lacombe J, et al. Scl regulates the quiescence and the long-term competence of hematopoietic stem cells. Blood. 2010;115(4):792-803.

67. Reynaud D, et al. SCL/TAL1 expression level regulates human hematopoietic stem cell self-renewal and engraftment. Blood. 2005;106(7):2318-2328.

68. Rojas-Sutterlin S, Lecuyer E, Hoang T. Kit and Scl regulation of hematopoietic stem cells. Curr Opin Hematol. 2014;21(4):256-264.

69. Souroullas GP, Salmon JM, Sablitzky F, Curtis DJ, Goodell MA. Adult hematopoietic stem and progenitor cells require either Lyl1 or Scl for survival. Cell Stem Cell. 2009;4(2):180-186.

70. McCormack MP, et al. Requirement for Lyl1 in a model of Lmo2-driven early T-cell precursor ALL. Blood. 2013;122(12):2093-2103.

71. Wilson A, et al. c-Myc controls the balance between hematopoietic stem cell self-renewal and differentiation. Genes Dev. 2004;18(22):2747-2763.

72. Escuin D, et al. The hematopoietic-specific beta1tubulin is naturally resistant to 2-methoxyestradiol and protects patients from drug-induced myelosuppression. Cell Cycle. 2009;8(23):3914-3924.

73. Hutchinson L, Kirk R. High drug attrition rates where are we going wrong?. Nat Rev Clin Oncol. 2011;8(4):189-190.

74. Xia B, et al. c-Myc plays part in drug resistance mediated by bone marrow stromal cells in acute myeloid leukemia. Leuk Res. 2015;39(1):92-99.
75. Hartwell KA, et al. Niche-based screening identifies small-molecule inhibitors of leukemia stem cells. Nat Chem Biol. 2013;9(12):840-848.

76. Chattopadhyay S, et al. Niche-based screening in multiple myeloma identifies a kinesin- 5 inhibitor with improved selectivity over hematopoietic progenitors. Cell Rep. 2015;10(4):755-770.

77. Kenny HA, et al. Quantitative high throughput screening using a primary human three-dimensional organotypic culture predicts in vivo efficacy. Nat Commun. 2015;6:6220.

78. Itoh K, et al. Reproducible establishment of hemopoietic supportive stromal cell lines from murine bone marrow. Exp Hematol. 1989;17(2):145-153. 